\title{
The first dozen years of the history of ITEP Theoretical Physics Laboratory
}

\author{
B.L.Ioffe \\ A.I.Alikhanov Institute of Theoretical and Experimental Physics (ITEP), \\ B.Cheremushkinskaya 25, 117218, Moscow, Russia
}

\begin{abstract}
The theoretical investigations at ITEP in the years 1945-1958 are reviewed. There are exposed the most important theoretical results, obtained in the following branches of physics: 1) the theory of nuclear reactors on thermal neutrons; 2) the hydrogen bomb project ("Tube" in USSR and "Classical Super" in USA); 3) radiation theory; 4) low temperature physics; 5) quantum electrodynamics and quantum field theories; 6) parity violation in weak interactions, the theory of $\beta$-decay and other weak processes; 7 ) strong interaction and nuclear physics. To the review are added the English translations of few papers, originally published in Russian, but unknown (or almost unknown) to Western readers.
\end{abstract}




\section{How I became a theoretical physicist}

In the war time, when the German forces came close to Moscow, I with my mother were evacuated from Moscow to Siberia (my father was in the army) and came back in June of 1943. I was 17 years old and had finished 9 classes of the middle school. (The middle school had 10 classes.) The question arose for me: what to do next? To continue learning in the school made no sense: next year I would be 18 and should go to the army. And such youths as I was, having no experience and not adapted to army life, when they appear at the battle front, as a rule, perished in the first attack. (I new this well, since in 1942 for half a year, I was working in a military hospital.)

At that time, in the summer of 1943, preparatory classes were open in several Moscow technical colleges (in USSR they were called Institutes), where the students were taught engineering specialities. These classes accepted pupils who had finished 9 classes of middle school. For few months they studied the program of 10-th class and after that became students of the college. The students of such colleges should not go to the army: even in the year 1943 the goverment understood that after the war the country would need engineers. (The main part of old engineers were killed in the battles.) There were no preparatory classes in Moscow University, and at energy and aviation colleges the deadline for acceptions had passed. The only college where it was still possible to enter preparatory classes was the Moscow Electro-Mechanical Institute of Railway Transport Engineers (MEMIIT). I became its student and in 1944 finished the 1-st course.

After the 1-st course, in the summer of 1944, students of MEMIIT were sent wood logging for a month to a village near the Moscow-Volga Canal. We, four boys, were settled in a small room of a peasant house. We were prepared for what would happen and had taken with us powder against the bugs, and strew the floor and the walls up to one meter height. At night, when we woke up and lit a lantern, we found that all the walls above this line were mowing - they were occupied by bugs. Then it became clear, that there were no toilets in the whole village - $100 \mathrm{~km}$ from Moscow we were in the Middle Ages.

I was not enthusiastic about the prospect of being a railway engineer, I wanted to be a physicist. I decided to enter the external department of Moscow University, where tuition was proceeding by correspondence. To do that, I needed permission from MEMIIT, but I got that easily since I had a good reputation there. Moreover, I was given the right to visit lectures freely (not obligatory), which was a very rare case. I entered the correspondence division of the Physical Faculty in autumn of 1944 and successfully passed all exams in the winter session - I got the highest marks in all disciplines. In March-April 1945 a new enrollment was announced for students in a special group at the Physical Faculty. In this group students of other institutes were admitted without any exams. If the student was enlisted in such group, his former institute had no right to keep him anymore and such person was free from the conscription to the army. Now it is clear that it was the enrollment for the atomic project. I stress that such enrollment proceeded earlier than the explosion of the first atoming bomb. I tried to enter this special group but was rejected, although I had an advantage in comparison with other students - I had passed the first examination session at the Physical Faculty. David Kirzhnits (a future corresponding member of the Russian Academy of Sciences) was also rejected, although he had a recommendation from Landau.

It is interesting how he got this recommendation. Kirzhnits was a student at MAI (Moscow Aviation Institute). The teacher of physics (a women) noticed his nonordinary abilities. She knew Landau and told him about this talented student. Landau invited Kirzhnits for a conversation. After the conversation Landau said: "I will write a letter of 
recommendation to Predvoditelev - the dean of the University Physical Faculty." He took a sheet of paper and a pen, sat down and started to think. "I cannot write "Dorogoi Alexander Savvich" - said Landau (in Russian "dorogoi" is the standard form of addressing somebody in a letter but it also means that the addressee is dear to the author of the letter) - He is not dear to me. I cannot write "Uvazhaemyi .." (respected): I have no respect for him". He thought a little more, then exclaimed: "Oh, I will write him: "Dear" - in English "dear" has no definite meaning. The reason of rejection of Kirzhnits and me was our nationality antisemitism was on the rise.

In the spring I should sit the exams at MEMIIT and at the correspondence division of the Physical Faculty of the University. I decided to get a bare pass mark in the exams on railway disciplines in MEMIIT so that it would be easier to leave MEMIIT. One of the courses was: "Fuel, water and lubricant." I had not studied it, and looked through the textbook only for a few hours before the exam. The examiner asked me: "There is a mixture of water with kerosine. How do you separate them?" I could not give any answer besides: to leave the mixture to stay for some time, kerosine will be above, the water below. I got a 5 - the highest mark.

I passed all exams at the correspondence division of the Physical Faculty with mark 5 and received very good recommendations from university professors Gradshtein and Modenov. Starting from autumn I again began attempts to transfer from the correspondence division to the normal Physical Faculty. I did not mention at the University that I am a student of MEMIIT, otherwise my request would have been rejected.

I had to visit the same deputy dean of the Physical Faculty, Georgi Petrovich Z., who had rejected me when I tried to enter the special group. The result was the same - he said: the transfer from the correspondence division to the normal faculty is forbidden. Then I visited the vice-rector of Moscow University Prof. Spizyn. He said the same, but added symphatetically: "You can complain, it can be repeated infinitely." "And where to complain ?" - asked I. "In the Ministry of Education" - was the answer.

I went to the Ministry of Education, to the Head of University Department. And here I had luck! The Head of Department Zatsepin was a decent person, he said: "You want to transfer from the correspondence division to the Physical Faculty ? Please do it." "And you can sign a letter, that you don't object ?" - asked I. "Yes, sure" - he answered. The letter was prepared and signed in a few minutes. With this letter I went to the Physical Faculty and here I had luck again! Georgi Petrovich was on vacation and instead of him was another man. Looking at him with innocent eyes, I said: "Georgi Petrovich promised me that, if I bring a letter from the Ministry of Education, saying that the Ministry do not object to my transfer from the correspondence division to the Physical Faculty, then he would put me in the list of students. Here is such letter." "I will do the order" - said the man. When Georgi Petrovich came back, he could do nothing: I already was a student. But I had to leave MEMIIT and leave without a scandal: overwise my documents would be sent to military committee and I would be called to the army although the war was over. I succeeded doing this after 7 visits to the dean and rector of MEMIIT.

So, I became a physicist. But a lot of time passed till the hope appeared that I can be a theorist. For a long time I hesitated, and finally decided to take Landau's "theoretical minimum" examination. The first exams - the entrance exam on mathematics, the exams on mechanics, classical field theory and the first part of statistics - I passed relatively easily. But in the study of quantum mechanics some problems were unclear to me: they had to be studied by reading original papers because the Landau-Lifshitz textbook did not yet exist at that time. 
At the 4-th course the students were distributed among the various departments - cathedras in Russian. I applied to the cathedra of theoretical physics and was accepted. But soon a new order came and I was transferred to the cathedra "The structure of matter". This was a coded name; in fact it meant the cathedra of nuclear and elementary particle physics. I was disappointed by such a decision and tried to have it revoked, but later I understood that again I had luck. The matter was, that at this cathedra the diploma supervisor could be any person participating in the atomic project, while in other cathedras the supervisor had to be a faculty professor.

Kirzhnits and I wanted to have a supervisor from the Landau school. We succeeded to get two phone numbers - Pomeranchuk's and Kompaneetz. Kirzhnits started calling Pomeranchuk, tried a few times, but unsuccessful - Pomeranchuk was absent. Then he called Kompaneetz and Kompaneetz agreed to be his supervisor. So, what was left for me - to call Pomeranchuk. I did many such calls and got the same answer: "Pomeranchuk is not here." Later, when I started to work at ITEP, I understood, what was the matter. The telephone of which I had the number, was not in Pomeranchuk's office but in the hall, where a soldier was sitting. Finally, - oh miracle - the same voice asked Pomeranchuk to the phone. Probably, at this moment Pomeranchuk just happened to cross the hall or had a conversation with somebody in the hall. Again, I had luck! I told Pomeranchuk, that I was a student at the university, that I had 3 courses of the Landau minimum and I was asking him to be my diploma supervisor. For Pomeranchuk, the fact, that I had passed 3 courses of the theoretical minimum was in my favour (at that time not so many people passed the minimum - about 10 persons) and he invited me to visit him for a final conversation. The day when I came was very cold. I had no fur coat nor even a warm coat - I was in a short pilot jacket, sent to USSR by the US within the framework of the lend-lease program. This jacket determined my fate - Pomeranchuk agreed - he told me later, that the jacket played the role of the last straw.

Pomeranchuk helped me very much with the preparation for the exam on quantum mechanics: he gave me the proofs of those chapters of "Quantum mechanics", where the problems were expounded that were unclear to me. After that I passed quantum mechanics as well as a few other courses. I began my diploma work, the theme of which was given to me by Pomeranchuk. But I was not sure whether I would succeed in becoming a theoretical physicist. Moreover, I had not much hope to achieve it. As was said once of the heroes of Sinclair Lewis' book "Arrowsmith": "Not everybody working in science is a scientist. Only few of them are." Even more this refers to theoretical physics: one thing is to pass exams and quite another is to work creatively. The first time that hope, that I have chances to be a theoretical physicist appeared at me during the work on the problem put to me by Pomeranchuk. I clearly remember this moment - the starry moment in my life.

Pomeranchuk suggested to me to calculate the polarization of slow (resonance) neutrons scattering on nuclei. This polarization arises due to interference of the nuclear scattering amplitude with the amplitude of the neutron magnetic moment interacting with the nuclear Coulomb field (relativistic effect). The amplitude of interaction of neutron magnetic field with Coulomb field is purely imaginary. Therefore interference is possible only if the nuclear scattering amplitude has an imaginary part. The similar problem was solved previously by Schwinger, who considered neutron scattering at high energies, where the nuclear amplitude is caused by diffraction and is pure imaginary. In the problem considered by Schwinger, the momentum transfers were much greater than the inverse sizes of the atom and the interaction of the neutron magnetic moment with atomic electrons could be neglected. Pomeranchuk suggested to me to consider neutron scattering at small energies in the domain of resonances, 
where the nuclear amplitude also has an imaginary part. Here, however, the momentum transfers are comparable with inverse atomic sizes. I tried to find the domain, where it would be possible to apply Schwinger's method and not consider the interaction of the neutron magnetic moment with atomic electrons, but the results were inconclusive and I was in the dumps. And suddenly, sitting in the University library - I clearly remember this moment - I realized, that I can perform the calculation exactly: it is necessary to take account of the atomic form factor, and I can do this by the Thomas-Fermi quasi-classical approach. It was my idea, Schwinger did not have it! And there, the first time, appeared the hope that I could become a theoretical physicist.

Besides this problem, I solved during my diploma practice two other problems which Pomeranchuk gave me: the calculation of photon polarization by bremsstrahlung and the production of $e^{+} e^{-}$pairs by polarized photons on a nuclei. At that time there was no Feynman technique, so I used the old, very complicated (Heitler) technique, where the states of electrons with positive and negative energies were treated separately. I did not published these calculations because I considered them as a technical exercise. Later I came to regret this strongly, because after a few years several groups published such calculations. (A short resume of these papers was published much later [1].)

\section{The foundation of ITEP and its program}

ITEP was organized by decree of the USSR government on December 1, 1945. (At that time it was called Laboratory No. 3 of USSR Academy of Sciences.) Lab. No. 3 was the second, after Lab. No. 2 (now Kurchatov Institute), organized within the framework of the Soviet Atomic Project. In fact Lab. No. 3 did not belong to the Academy of Sciences, but to the First Directorate of the Council of Ministers of the USSR, which was responsible for the atomic project. The problems, which should be investigated by ITEP were formulated as:

- physical investigation, design and construction of nuclear uranium reactor with heavy water as moderator;

- physical investigations of thorium-heavy water and thorium-uranium-heavy water systems for production of uranium -233;

- physical investigations of $\beta$-radioactivity;

- physical investigations of high energy nuclear particles and cosmic rays.

ITEP theoreticians participated in the investigation of all these problems, but the field of their interest was much broader. For the first half of 1946 the Head of the ITEP Laboratory of Theoretical Physics was L.D. Landau, after that I.Ya.Pomeranchuk took this place, but Landau was continuing working in the Theoretical Laboratory and came to ITEP regularly every week till 1958.

\section{The theory of nuclear reactors on thermal neutrons}

The theory of nuclear reactors began with a famous paper by Zeldovich and Khariton [2], published in 1940. They introduced in the theory the multiplication coefficient $k$, defined as the ratio of the number of neutrons produced in the fission to the number of absorbed neutrons in an infinite system, when the loss of neutrons due to escape from the system can be neglected. For $k$ was obtained the formula

$$
k=\nu^{\prime} \varphi \theta \varepsilon,
$$




$$
\nu^{\prime}=\nu \frac{\sigma_{f}}{\sigma_{f}+\sigma_{c}},
$$

where $\nu$ is the number of neutrons produced in the process of fission by absorption of a thermal neutron, $\sigma_{f}$ is the fission cross section, $\sigma_{c}$ is the absorption cross section without fission by fissionable material. (All cross sections refer to thermal neutrons; only one fissionable isotope is considered.) In (11) $\varphi$ is the probability of a fast (fission) neutron to reach thermal energies, escaping the resonance absorption in the course of moderation; $\theta$ is the probability of absorption of a thermal neutron by a fissionable nucleus (for definiteness let us speak about ${ }^{235} U$ ), but not by another nucleus; $\varepsilon$ is the probability for a fast neutron to perform additional fission in the same uranium slab. (The factor $\varepsilon$ was absent in [2], it was added later by G. Flerov.) The necessary condition for realization of a chain reaction is:

$$
k>1
$$

It is easy to find the condition for the reactor to be a breeder, i.e. to produce more fissionable material than is burned. Let $\nu^{\prime}$ neutrons be produced in the fission. Among them one neutron must be absorbed by a fissionable nucleus in order to have a chain reaction. One neutron should be absorbed by some material $\left({ }^{238} \mathrm{U}\right.$ or $\left.\mathrm{Th}\right)$ in order to restore the number of fissionable nuclei. So, the necessary condition for realization of a breeder is:

$$
\nu^{\prime}>2
$$

This condition is fulfilled for fast neutrons in the cycle ${ }^{238} U-P u\left(\nu^{\prime} \approx 2.9\right)$ and for thermal neutrons in the cycle $T h-{ }^{233} U\left(\nu^{\prime} \approx 2.3\right)$.

The most complicated problem in calculating the multiplicity coefficient $k$ is the calculation of $\varphi$ - the probability of the neutron to escape resonance absorption in the process of moderation. This problem was solved by Gurevich and Pomeranchuk in 1943 [3]. The basic points of their discussion are the following. Consider a separate resonance at low neutron energy (e.g. the first level of ${ }^{238} U$ at $E_{r}=6.67 \mathrm{eV}$ of width $\Gamma=25 \cdot 10^{-3} \mathrm{eV}$ and peak absorption cross section $\sigma_{0}=22 \cdot 10^{3}$ barn). Define [3] "the width of the dangerous zone" $\Delta E_{r}$ by the equality

$$
\sigma_{a}\left(E_{r}+\frac{1}{2} \Delta E_{r}\right) \rho \bar{l}=1
$$

where $\sigma_{a}(E)$ is the absorption cross section, $E$ is the neutron energy, $\rho$ is the number of absorbing nuclei per $\mathrm{cm}^{3}$ of the slab, and $\bar{l}$ is the mean path of the neutron in the slab. Assume that in the vicinity of the resonance $\sigma_{a}(E)$ is described by the Breit-Wigner formula

$$
\sigma_{a}(E)=\sigma_{a}\left(E_{r}\right) \sqrt{\frac{E_{r}}{E}}\left(1+x^{2}\right)^{-1}, \quad x=\frac{2}{\Gamma}\left(E-E_{r}\right)
$$

The substitution of ([6) in (5) gives

$$
\Delta E_{r}=\Gamma \sqrt{a}, \quad a=\sigma_{a}\left(E_{r}\right) \rho \bar{l}
$$

For the first, most important level in ${ }^{238} U$ we have $a \gg 1$ and $\Delta E_{r} \gg \Gamma$. This means that the main absorption of neutrons proceeds not in the central part of the resonance, but in the tales of the resonance curve. (Because of the very large cross section at the center of the resonance, absorption takes place in the periphery of the slab, strongly shielding its central part.) If we devide the resonance levels into two groups: low energy and high energy resonances, then the discussion presented above is valid for the former group. For the latter 
group the resonance absorption is simply proportional to the number of absorbing nuclei. Using the Breit-Wigner cross section formula for the neutron absorption in the resonance domain, it is possible to show that for the former group of levels the resonance absorption is proportional to $\pi r^{2} / \sqrt{\bar{l}}$, where $r$ is the slab radius. The final formula for resonance absorption found in 3 is

$$
\begin{gathered}
-\ln \varphi=\frac{\lambda_{s}}{\xi} \cdot \frac{\alpha d^{3 / 2}+\beta d^{2}}{a^{2}-\pi d^{2} / 4} \\
\alpha=\frac{\pi}{4} 0.975 \sqrt{\pi \rho} \sum_{i} \frac{\sqrt{\sigma_{a i}\left(E_{r}^{i}\right) \Gamma_{i}^{r}}}{E_{r}^{i}}, \quad \beta=\frac{\pi}{4} \rho \int_{E_{t h}}^{E_{f}} \sigma(E) \frac{d E}{E},
\end{gathered}
$$

where $\lambda_{s}$ is the scattering length in the moderator, $\xi$ is the mean logarithmic loss of energy in the moderator, $1 / \xi \approx A / 2+1 / 3$. In the derivation of $(8)$ it was assumed, that the generation of resonance neutrons in the moderator is homogeneous in space and isotropic in velocities. The result (8) is valid for small slabs, where $\bar{l}<\lambda_{s}$.

In the US the resonance absorption of neutrons was considered by E. Wigner, who proposed the interpolation formula

$$
-\ln \varphi=\frac{\lambda_{s}}{\xi} \frac{A d+B d^{2}}{a^{2}-\pi d^{2} / 4}
$$

In the first stage of atomic projects in the US and USSR, when the ${ }^{238} U$ level parameters were not well known, the constants in (8) and (10) were determined empirically by fitting the data and the fit was satisfactory for both formulae (8) and (10). But even in this case Eq. (8) has an advantage compared with (10): 1) It gives the Doppler broadening, and hence the temperature dependence could be predicted 1; 2) the case could be considered, when the slab contains the moderator, e.g. when the slab has the shape of a tube with moderator inside (such a calculation was done by A.Rudik at ITEP [4]).

In 1947 Akhiezer and Pomeranchuk wrote the book "The Introduction in theory of neutron multiplying system (reactors)" [5], where a detailed exposition of nuclear reactor theory was presented. Besides the theory of resonance absorption they considered the determination of reactor critical size, several aspects of the theory of heterogeneous reactors, reactor kinetics and temperature coefficient, solved exactly the boundary condition on a flat boundary of vacuum with medium (by solving the Boltzmann equation by the Hopf-Wiener method). The book was classified and was published only in 2002. The theory of heterogeneous reactors was based on a suggestion formulated by Landau: each slab is characterized by a constant $\eta$, equal to the ratio of the flux of thermal neutrons on its surface to the neutron density there. It was the first book on the theory of nuclear reactors in the world literature, the corresponding books in the USA appeared later.

Other members of the ITEP Theoretical Laboratory also made remarkable contributions to the theory of nuclear reactors: A.Galanin developed the theory of heterogeneous reactors [6], [7]; A.Galanin and B.Ioffe performed calculations of the $T h-{ }^{233} U$ cycle in heavy water reactors and demonstrated the principal realization of the breeder in such device [8]: B.Ioffe and L.Okun studied the deep burning of fuel in heavy water reactors (atomic power stations) [9]. In [9] it was shown (based on a suggestion by A.I. Alikhanov), that the continuous unload

\footnotetext{
${ }^{1}$ In some cases (e.g. the Chernobyl accident) the temperature dependence of the multiplication coefficient is extremely important.
} 
of slabs from the reactor has a serious advantage in comparison with simultaneous unload of all slabs - the burn-up of fuel increases by up to a factor 1.5, which is extremely important for atomic power stations.

On the basis of these theoretical investigations the first Soviet research reactor on natural uranium with heavy water moderator and cooling was built in 1949 in ITEP. The critical experiments performed at the start-up of the reactor demonstrated very good agreement of the theory with experiment. In 1955 the reactor was modernized and natural uranium was changed to enriched uranium. At the start-up of this reactor an accident happened which will be described in the next Section. Research reactors of this type were built also under scientific guidance by ITEP in China and Yugoslavia. According to ITEP physical and engineering design several heavy water moderated and cooled reactors for plutonium production were constructed in the USSR.

In 1972 the first atomic power station in Czechoslovakia - in Yaslovsky Bohunize (Slovakia) - was put in operation, using natural uranium fuel, heavy water moderator and gas cooling. Scientific guidance of the project was performed by ITEP, the physical design was done by Theoretical Laboratory members Ioffe and Iljichev. The critical experiment performed at the start-up and the series of experiments done after the startup demonstrated excellent agreement of theory with the data [10].

\section{Prevention of a dangerous accident at the ITEP re- actor in 1955}

The ITEP heavy water research reactor was reconstructed in 1955. Instead of natural uranium, the modernized reactor was to use $2 \%$ enriched uranium, all uranium slabs were replaced by ring-shaped ones, some construction elements were to be changed also. As a result the reactor power should increase 4-fold and the flux of thermal neutrons by one order of magnitude. I performed the physical design of the reactor. It was the first reactor, going into operation, for whose physical design I was completely responsible. (Previously I did only the calculations of the physics for projects of reactors which were not actually constructed. A.D.Galanin was the person, responsible for physical design of constructed reactors, and I was only an executor.)

So, come the day of physical start-up of the reactor, when the reactor should become critical. The head of ITEP reactor division and also the person responsible for the physical start-up of the reactor, S.Ya. Nikitin invited me to be present at the experiment.

The physical start-up of a heavy water reactor proceeds in the following way. The uranium slabs are inserted in the reactor, in which there is no moderator - no heavy water. Since there is no moderator, no chain reaction is going and there is no neutron flux. Then heavy water is poured in gradually. At some level of heavy water the reactor becomes critical, the chain reaction begins - the reactor starts to work. The critical level of heavy water - predicted by the theoretical design - is the main parameter for the future reactor exploitation. The coincidence of its experimental value with the theoretical prediction means that the theory is reliable enough and that the future exploitation of the reactor can proceed based on theoretical predictions. In case of contradiction of the theory with experiment any surprises are possible.

Before the beginning of the experiment Nikitin asked me what the theoretical prediction for the critical level and its accuracy was. I said that the critical level was $150 \mathrm{~cm}$ and that the error could not exceed $5 \mathrm{~cm}$. The pouring in of the heavy water began. Simultaneously 
the neutron flux $\mathrm{N}$ was measured in several places of the reactor (there was an artificial neutron source at the bottom of the reactor vessel). The dependence of $1 / \mathrm{N}$ as a function of the moderator level was plotted. Evidently, when criticality is achieved ( $\mathrm{N}$ turns to infinity), the curve $1 / \mathrm{N}$ must cross the abscissa. The level became $10 \mathrm{~cm}$ below my predicted value, then $5 \mathrm{~cm}$ below, but the curve $1 / \mathrm{N}$ did not look like going to the expected point. Nikitin consols me: Sometimes it happens, that the curve bends in the last moment. The predicted critical level was reached, but the reactor did not become critical. The predicted level was exceeded by $5-8 \mathrm{~cm}$, but the reactor did not start to work. It was easy to understand the thoughts in the minds of all people attending by looking at their faces: "The first reactor was calculated by Pomeranchuk and Galanin, and see what is happening when this responsible work is entrusted to young people". A few more centimeters of heavy water were added but nothing happened. At this point Nikitin gave the command to stop the experiment and reported about what had happened to Abram Isaakovich Alikhanov.

Alikhanov was strongly discontented - it was greatly annoying for him. It is possible, that for a moment he had the same thought in his mind as the experimentalists. However, he postponed all work, related to the start-up of the reactor to the next day and said to me: "Please check your calculations once more and report to me the results tomorrow". The whole evening I checked my calculations but did not find any mistakes. I did not sleep all night. In the morning I collected all my courage, came to Alikhanov and said: "I do not see any mistakes in the theoretical calculation. There should be no such large discrepancy between theory and experiment." Abram Isaakovich was impressed by my words and ordered: "Do not perform the setting up of the reactor operation, let the engineers search for mistakes".

So, two days passed. Then B.A.Medjibovski came to my office. He was an engineer, who was working on the reactor control system and had nothing to do with reactor construction and assembly. He asked me: If uranium slabs were fixed not at their right positions, but 20 centimeters higher, what would be the critical level ? I performed the simple calculation and answered: "Just at the point where the trend of the 1/N curve was!" Medjibovski explained that he had found a place on the blueprints, where by mistake the ends of the slabs could be fixed, very similar to the right one but 20 centimeters higher. He immediately went to tell his guess to Nikitin. Nikitin called A.P.Shilov, the senior mechanic, the person, responsible for assembling the reactor. Shilov began to shout: "Nonsense! This could not happen ! Never!" Then Nikitin ordered to take off the upper cover of the reactor, said that next day he would measure himself in what position the uranium slabs were fixed and invited me to assist at that. When I came, the upper cover of the reactor was taken off, Nikitin was standing above. He was in dark goggles, gloves and overalls. Probably something was put on under the overall. It must be mentioned, that it is not safe to stay above the reactor when the upper cover is taken off. Although the reactor was not working, there was still some neutron flux with associated radiation. Therefore all people should stay at a large distance from the reactor. Nikitin took a long stick, put it in the reactor, marked some point on the stick, took it out and measured its length up to the mark. This procedure he repeated at several places of the reactor. Then he announced: "The slabs are fixed at the wrong places: 20 centimeter higher, than they should be. I will report this to Alikhanov". The reactor was reassembled. If the reactor had been put in operation with such a wrong assembly, then the upper ends of the uranium slabs would have been above the moderator level, which would result in a strong increase of radiation caused by fast neutrons, and the consequence would have been very undesirable. (It must be recalled, that ITEP is situated in the city of Moscow and the distance from the reactor to the nearest houses is only about a few hundred meters.) 


\section{The hydrogen bomb project ("Tube" in USSR and "Classical Super" in USA)}

There are two fusion reactions which can be used for the realization of a hydrogen bomb:

$$
\begin{aligned}
& D+D \rightarrow{ }^{4} \mathrm{He}+\gamma+23.8 \mathrm{MeV} \\
& D+T \rightarrow{ }^{4} \mathrm{He}+n+17.6 \mathrm{MeV}
\end{aligned}
$$

At low energies the cross section of the latter reaction is about two orders of magnitude greater than that of the former one and the process (12) proceeds at lower temperatures, than that of (11). But tritium is unstable (its half life is 12 years), does not exist in nature and can be produced only in nuclear reactors in rather small amounts. In 1941 Fermi in a conversation with Teller put forward the idea, how the process (11) can be used for the realization of an H-bomb [11. Suppose that there is a long tube filled with liquid deuterium. At one end of the tube there is an igniter - an atomic bomb. When the atomic bomb explodes a shock wave propagates along the tube, compressing the deuterium and strongly increasing its temperature: the thermonuclear reaction starts. It must be stressed that the length of the tube is not restricted. Therefore the power of the bomb is also unlimited. Teller was encouraged by this idea, and began to work on its realization with his group. In Soviet Union the corresponding project was presented by Gurevich, Zeldovich, Khariton and Pomeranchuk on 17.12.1945 [12. I have doubts, that the idea of the Soviet project was original - probably it came from intelligence service, but I believe that the concrete and detailed calculations performed by the authors of the Russian project were original (see [13], [14]). The possibility of realizing the project is determined by energy balance: if it is positive, i.e. the energy release by nuclear reactions is greater than the energy loss through the surface of the tube, than the shock wave will propagate along the tube and the hydrogen bomb explodes, if not, the reaction will dump. The main source of energy loss from the system are the $\gamma$-quanta produced by electron bremsstrahlung. The $\gamma$ 's can undergo Compton scattering on electrons. Since the spectrum of $\gamma$ 's produced by bremsstrahlung is softer than the spectrum of electrons, in Compton scattering $\gamma$-quanta increase their energy in the mean, unlike the case of usual Compton effect on electrons at rest. (This process can be called inverse Compton-effect.) As a result of the inverse Compton effect the loss of energy from the system is increasing.

Before 1949 no more calculations were done in USSR on the project, which got the jargon name "Tube", since there was no atomic bomb in the USSR - the igniter, needed for its realization. In 1949 the calculations resumed. They were performed by Zeldovich's group at Arzamas-16 (now Sarov). Their goal was to calculate the energy balance. To do this it was necessary to know the comptonization coefficient, defined as the ratio of the energy, carried by $\gamma$ 's outside the tube after (multiple) inverse Compton collisions, to the energy of bremsstrahlung. The result of the calculation was, that the balance is zero. But the accuracy of calculations was low: the accuracy of the comptonization coefficient calculation was given by a factor of 1.5 or even 2. (E.g. the Compton cross section integrated over angles was used, whereas this cross section significantly depends on angles.) To increase the accuracy to $10 \%$ requires much more refined methods: taking account of relativistic effects, anisotropy etc. At that time (in 1951) Pomeranchuk was sent to Arzamas-16 by a high level decision for a long term visit. Pomeranchuk was overburdened by his stay there. It was the time of fast progress in quantum electrodynamics and Pomeranchuk wanted to participate in this work, but this was impossible in Arzamas-16. So, he suggested that he and his group 
would do the calculations with higher accuracy, up to $10 \%$, if he would be allowed to go back to ITEP. He had reasons for such suggestion: at ITEP we were familiar with the Feynman technique, which was necessary for relativistic calculations, and at ITEP there was a strong mathematical group headed by the high class methematician A.Kronrod, who liked numerical calculations needed in this problem. The Pomeranchuk proposal was accepted, he returned to Moscow and started to form the group. Unfortunately, I became the only physicist actively participating in the solution of this problem. My colleague A.Rudik did not get permission to participate although he was taking part in all calculations of reactors, including the ones for plutonium and tritium production. The H-bomb project had the highest degree of secrecy, and he was not allowed to this level (why this happened is an enigma to me even now.) Galanin was entirely busy by reactor calculations, Berestetsky participated in the solution of particular problems, separated from the main one. So, I was left alone. But our group got strong support for numerical calculations. M.Keldysh, Head of the Committee for mathematical provision of the Atomic Project, got at our disposal a group of 40 ladies from Leningrad, who performed calculations on electro-mechanical calculators. This group was headed by L.Kantorovich, specialist in numerical calculations and future the Nobel prize laureate in economics. I calculated the inverse Compton scattering cross section on arbitrarily moving electrons and integrated it over the Maxwell spectrum of electrons at given temperature [15]. Kronrod invented an effective method of the numerical calculation: it was the solution of the Peierls equation along the line of flight of the $\gamma$-ray. The work proceeded in the following way: the Zeldovich group performed the hydrodynamical calculations and presented to us the distributions of $\gamma$ 's, electrons and heavy particles, we performed the calculations of the energy loss at each point and returned the results to them back, they perform new hydrodynamical calculations and so on. After 7 iterations the process converges. The result for energy balance was negative: if the energy, released in the nuclear reaction is to be taken as 1 , then the energy leaving the tube was equal to 1.2 . The system was not working, such H-bomb could not be realized in principle. Later it became known that Teller had come to the same conclusion for the "Classical Super".

Mankind was lucky, or maybe God had been merciful.

Since the energy balance was only slightly negative it was necessary to check the influence of various small effects which were neglected in the calculation. Among them was the influence of polarization of the $\gamma^{\prime}$ 's. All calculations had been performed using the Compton cross section averaged over polarization of initial and final photons. However, the effect of polarization could be significant. For example in Thomson scattering (the low energy limit of Compton scattering), if the initial photon is unpolarized, then the final photon has a significant polarization. Also, in the same limit, if the initial photon is completely polarized, then there arises a significant azimuthal asymmetry in the final photon distributions. The polarization does not affect the comptonization coefficient for small systems (less than the $\gamma$ 's scattering length), because the polarization can influence the angular distributions only if there are two or more collisions. On the other hand, the effect of polarization can be neglected in diffusion approximation, i.e. for large systems (this statement was proven in [16]). So, one may expect that the influence of polarization is small also in the intermediate case.

Photon polarization cannot be accounted for within the framework of classical theory - by solution of Boltzmann or Peierls equations. The photon polarization can take only two values, therefore it is a quantum object. For this reason it was necessary to consider a $2 \times 2$ density matrix. This was done in [16], where the following equation for the density matrix 
was obtained:

$$
\begin{gathered}
\frac{\partial J_{\mu \nu}(\mathbf{k}, \mathbf{r})}{\partial t}+\mathbf{n} \nabla J_{\mu \nu}(\mathbf{k}, \mathbf{r})+\frac{J_{\mu \nu}(\mathbf{k}, \mathbf{r})}{l(k)}=\int d \mathbf{k}^{\prime} d \mathbf{p}_{1} n\left(\mathbf{p}_{1}\right) W_{\mu \nu, \lambda \sigma}\left(k^{\prime}, k, p_{1}, p_{2}\right) \times \\
\times J_{\lambda \sigma}\left(\mathbf{k}^{\prime}, \mathbf{r}\right) \delta\left(k^{\prime}+E_{p_{1}}-k-E_{p_{2}}\right)+F_{\mu \nu},
\end{gathered}
$$

where $J_{\mu \nu}(\mathbf{k}, \mathbf{r})$ is the relativistic generalization of the density matrix for $\gamma^{\prime} \mathbf{s}, \mathbf{k}$ is the $\gamma^{\prime} \mathrm{s}$ momentum 3-vector, $\mathbf{n}=\mathbf{k} / k, n(p)$ is the Maxwell distribution of electrons, $F_{\mu \nu}$ is the bremsstrahlung source of $\gamma$ 's:

$$
F_{\mu \nu}(\mathbf{k}, \mathbf{r})=\frac{1}{2} Q(\mathbf{k}, \mathbf{r}) \delta_{\mu \nu}^{\perp}
$$

$Q(\mathbf{k}, \mathbf{r})$ represents the total number of gamma's, emitted per unit of time, $\delta_{\mu \nu}^{\perp}$ is the unit tensor with nonzero components perpendicular to $\mathbf{k}$, and $l(k)=1 / w(k)$. In the limit $k / m_{e} \ll 1$ the kernel $W_{\mu \nu \lambda \sigma}\left(k^{\prime}, k, p_{1}, p_{2}\right)$ is given by:

$$
\begin{gathered}
W_{\mu \nu \lambda \sigma}\left(k^{\prime}, k, p_{1}, p_{2}\right)=\frac{e^{2}}{4 E_{1} E_{2}} \frac{1}{k k^{\prime}}\left\{\delta_{\mu \lambda}-\frac{1}{\left(p_{1} k^{\prime}\right)}\left[p_{1 \lambda} k_{\mu}^{\prime}+p_{1 \mu} k_{\lambda}^{\prime}\right]+\frac{k k^{\prime}}{\left(p_{1} k^{\prime}\right)^{2}} p_{1 \mu} p_{1 \lambda}\right\} \times \\
\times\left\{\delta_{\nu \sigma}-\frac{1}{\left(p_{1} k^{\prime}\right)}\left[p_{1 \sigma} k_{\nu}^{\prime}+p_{1 \nu} k_{\sigma}\right]+\frac{k k^{\prime}}{\left(p_{1} k^{\prime}\right)^{2}} p_{1 \nu} p_{1 \sigma}\right\}+(\mu \rightarrow \nu, \lambda \rightarrow \sigma) .
\end{gathered}
$$

(The exact expression for $W_{\mu \nu \lambda \sigma}$ was presented in [16].) Equation (13) was solved in [16] in Thomson limit for the case of a plate of thickness of the order of 2-3 scattering lengths. It was found that taking account of polarization for 2 collisions decreases the yield of photons by by $1-2 \%$. That means, that the number of Compton scatterings increases, if the polarization is taken into account. The conclusion was: taking account of polarization gives of the order of a few percent in the comptonization coefficient and, probably, results in its increase. So, this statement strengthens the general conclusion about the impossibility of realization of such a bomb. From the theoretical point of view it was the first time that the Boltzmann equation was written and solved for a quantum system.

In connection with this problem an episode happened which I would like to mention here. One day, when this work was approaching its end, Pomeranchuk came to the office occupied by Rudik and myself. He said: "Soon both of you should present your doctoral theses. The subject of Rudik's thesis can be declassified, but yours - he said to me - must be classified". (I remind the reader, that this happened in the autumn of 1952, when the wave of antisemitism was growing higher and higher, the "doctors affair" was ahead; Rudik was Russian, I am a Jew.) I presented as my thesis: "The influence of polarization on $\gamma$-quanta propagation in ionized gas". Although the purpose of the calculation was not mentioned in the thesis, moreover the words "thermonuclear reaction" were not mentioned, it had the highest level of secrecy: top secret, special folder. The approval of classified theses at that time could be given at only one place: the Scientific Council of the Kurchatov Institute. The Chairman of the Council was Kurchatov, Vice-Chairman was Artsimovich. After I had presented my thesis and the opponents - I.Tamm and I.Khalatnikov - had given their positive response, one member of the Council stood up and said: "O'key, I have no objection. But I have a question. I do not understand why this thesis has such high level of secrecy ?" And Artsimovich, who chaired the meeting, replied: "And it is quite good that you do not understand!" 
Besides the solution of this problem, V.Berestetsky, I.Pomeranchuk and myself performed in 1951 another calculation ordered by Zeldovich: The calculation of heat conduction of completely ionized gas at high temperatures [17]. This calculation also required a fully relativistic treatment: the Boltzmann equation for electron distribution function must be written in relativistic form. Besides the temperature gradient, the external electric field $\mathbf{E}$ had to be accounted for. The electric field $\mathbf{E}$ arises because of redistribution of charges in the gas, which happens before the equilibration of temperatures. The value of $\mathbf{E}$ is determined from the requirement of the vanishing of the total electric current.

The main idea of the calculation was based on the known fact [18] that large perpendicular distances to the collision direction play the dominant role in the gas-kinetical processes in the case of Coulomb interaction. The importance of large impact parameters corresponds to small momentum transfers in electron-ion collisions. The results were obtained after tedious numerical calculations. I will not present here the whole set of results, but restrict myself to presenting the value of heat conductivity $\kappa$ in the ultrarelativistic case $\left(T \gg m c^{2}\right)$ :

$$
\begin{gathered}
\kappa=0.25 \frac{1}{L}\left(\frac{T}{e^{2}}\right)^{2} c \\
L=\frac{r_{\text {max }}}{r_{\text {min }}} \approx 10
\end{gathered}
$$

where $r_{\max }$ and $r_{\min }$ are the maximal and minimal impact parameters. 2 .

\section{Radiation theory}

In 1939 Pomeranchuk started the study of radiation, emitted by electrons in magnetic fields. He demonstrated that because of radiation the energy of cosmic ray electrons falling on the Earth surface is limited, and in particular if the electrons are moving vertically in the plane of the magnetic equator, then their energy cannot exceed $4 \cdot 10^{17} \mathrm{eV}$ [19]. The same idea was exploited in [20]: there it was shown that in a betatron the energy of circularly orbiting electrons accelerated by the varying magnetic field inside the circle is limited by

$$
E_{0}=m c^{2} \sqrt{\frac{3}{2} \frac{e}{c} \frac{R_{0}}{r_{0}^{2} H^{2}}\left|\frac{d H}{d t}\right|},
$$

where $R_{0}$ is the radius of the orbit and $r_{0}=e^{2} / m c^{2}$. The angular distribution of the radiation was analyzed in [21] and it was shown that it was concentrated in a small region of angles relative to the plane of the orbit. The spectrum of the radiation is almost a constant up to $\left(E_{0} / m c^{2}\right)^{3} \omega_{0}$, where $\omega_{0}$ is the orbiting frequency and the spectrum is represented by equidistant lines. This radiation - called synchrotron radiation - is now intensively exploited in many fields: atomic and molecular physics, physics of condensed matter, biophysics etc. A specially interesting possibility arises when the electrons are moving in periodically varying magnetic field along the orbit (the undulator). When the electrons are concentrated in bunches and the number of electrons per bunch is large enough, then it becomes possible to construct a "laser on free electrons" (the undulator, surrounded be optical reflector), which can produce laser optical beams with frequencies from infra-red up to ultra-violet. The creation of free electron lasers opens a wide field of new possibilities in physics and technology.

\footnotetext{
${ }^{2}$ This work was done in 1951 and classified, declassified in 1972 and published in the Collected Works of Pomeranchuk [17].
} 
There is some analogy of the origin of synchrotron radiation and the deviation of the bremsstrahlung spectrum in matter from that given by the Bethe-Heitler formula (LandauPomeranchuk effect). Synchrotron radiation is caused by the curvature of the electron trajectory in a magnetic field, resulting in acceleration of the electrons. When an electron passes through matter it undergoes multiple scattering, which results in electron acceleration in the direction transverse to its line of flight. This acceleration induces radiation, which interferes with bremsstrahlung and influences the bremsstrahlung spectrum. Following Landau and Pomeranchuk, consider the case when the bremsstrahlung photon energy $\omega$ is much less than the electron energy $E, \omega \ll E$. In this case the classical theory of radiation is applicable.

Let us introduce the coherence length $l_{c}$ which, by order of magnitude, determines the longitudinal distance (along the electron momentum) where the bremsstrahlung photon is formed:

$$
l_{c}=\frac{E^{2}}{m^{2} \omega} .
$$

At high electron energies the angles of photon emission are small:

$$
\theta \sim \frac{m}{E}
$$

The mean square of the angle of multiple scattering of an electron travelling the distance $L$ in amorphous medium is given by

$$
\left\langle\vartheta^{2}\right\rangle_{L}^{2}=\frac{E_{s}^{2} L}{E^{2} L_{R}}
$$

where $E_{s}^{2} \approx 4 \pi m^{2} / e^{2}$, and $L_{R}$ is the radiation length

$$
L_{R}^{-1}=\frac{4 Z^{2} e^{6} n}{m^{2}} \ln \left(183 Z^{-1 / 2}\right)
$$

(here $n$ is the number of atoms per $\mathrm{cm}^{3}$ and $Z$ is the atomic number). Let us put $L=l_{c}$. If $\left\langle\vartheta^{2}\right\rangle_{l_{c}}$ exceeds $\theta^{2}$, then the influence of multiple scattering on bremsstrahlung radiation becomes important. In the case of condition $\left\langle\vartheta^{2}\right\rangle \gg \theta^{2}$, which is equivalent to $\omega \ll E^{2} / E_{0}$, $E_{0}=m^{2} L_{R} / E_{s}^{2}$, Landau and Pomeranchuk [22] obtained the formula for bremsstrahlung intensity, emitted per unit of time by electrons in matter:

$$
\frac{d I}{d \omega}=2 \sqrt{\frac{\omega m^{2} e^{2}}{\pi E^{2} L_{R}}}
$$

(In fact, Landau and Pomeranchuk performed the calculations, correct by order of magnitude. The formula presented above was obtained by Migdal [23], who succeeded in solving the problem exactly, see also [24].)

The number of quanta, emitted in the frequency interval $d \omega$ is given by

$$
d N=2 \frac{d \omega}{\sqrt{\omega}} \sqrt{\frac{m^{2} e^{2}}{\pi E^{2} L_{R}}}
$$

Since the condition $\omega \ll E^{2} / E_{0}$ is fulfilled at small $\omega$, eq.(24) states that there is no infrared catastrophe in matter: the total number of emitted quanta is finite. 


\section{Low temperature physics}

The best known work done at ITEP in low temperature physics is Pomeranchuk's investigation of the properties of ${ }^{3} \mathrm{He}$ [25]. At low temperature ${ }^{4} \mathrm{He}$ is a superfluid, which is caused by the Bose-Einstein statistics of ${ }^{4} \mathrm{He}$ atoms. ${ }^{3} \mathrm{He}$ nuclei have nuclear spin $1 / 2$, so that ${ }^{3} \mathrm{He}$ gas satisfies Fermi-Dirac statistics and the arguments which hold for ${ }^{4} \mathrm{He}$ are not valid for ${ }^{3} \mathrm{He}$. Pomeranchuk's considerations were the following. At low temperatures, when the wave lengths of ${ }^{3} \mathrm{He}$ atoms are of order of their distances, the interaction between two ${ }^{3} \mathrm{He}$ atoms depends on the orientation of their nuclear spins and exchange effects are important. As a result of such effects the antiparallel orientation of two neighboring nuclear spins is preferred. Indeed, in case of antiparallel orientation the coordinate wave function can correspond to $s$-wave, while in case of parallel orientation (the total spin equal to 1 ), it is a $p$-wave. In the former case the distance between two ${ }^{3} \mathrm{He}$ nuclei is smaller and the attractive interaction is stronger.

Let us compare the values of entropy in the solid (crystal) and liquid phases of ${ }^{3} \mathrm{He}$. In the solid phase, as was shown by Pomeranchuk, the amplitudes of the zero modes of oscillations are much smaller than the distances between the atoms. (The crystal state may be realized at high pressure). So, the spins of atoms are uncorrelated and the entropy is a constant equal to $s_{c r}=\ln 2$. The entropy in the crystal begins to go to zero at very low temperatures $T_{0}$. (Pomeranchuk estimated $T_{0}$ as $T_{0} \sim 10^{-7} \mathrm{~K}$; modern estimates give $T_{0} \sim 10^{-3} K[26]$.) In the liquid phase, since the spins are correlated, the entropy is going to zero proportionally to $T$ when $T$ decreases:

$$
s_{\text {liq }}=\left(T / T_{F}\right) \ln 2
$$

(See Fig.1). Therefore, at $T_{0}<T<T_{F}$ the entropy of the solid phase is greater than the entropy of the liquid phase - the situation is opposite to the standard one. At the isothermal melting the heat is secreted, not absorbed as usually.

In order to find the pressure- $T$-dependence along the phase transition curve, write the Clausius-Clapeyron relation

$$
\frac{d P}{d T}=\frac{s_{l i q}-s_{c r}}{v_{l i q}-v_{c r}},
$$

where $v_{l i q}$ and $v_{c r}$ are the volume per particle in the liquid and crystal, respectively. $v_{l i q}-v_{c r}$ is always positive. The dependence $P(T)$ is shown in Fig.2. (The dashed line corresponds to polarized ${ }^{3} \mathrm{He}$.) At the point $T_{m}$, defined by $s_{l i q}\left(T_{m}\right)=s_{c r}\left(T_{m}\right)$, the curve $P(T)$ has a minimum. Experimentally, $T_{m}=0.32 K, P_{m}=29$ bar. According to Pomeranchuk's theory it is possible to cool ${ }^{3} \mathrm{He}$ down to very low temperatures. The cooling proceeds in the following way. First reach the point $p_{m}, T_{m}$ by some or other method. Then adiabatically crystalize the liquid ${ }^{3} \mathrm{He}$ by increasing the pressure. This process will go along the dashed line on Fig.1 and solid line on Fig.2. During the process ${ }^{3} \mathrm{He}$ is first solidified and then again is melted.

In reality, as was found later, Pomeranchuk's reasoning was not completely correct $[26,27]$. The wrong assumption was that the distances between ${ }^{3} \mathrm{He}$ atoms are rather small; in fact in the tunnel exchange three or even more atoms of ${ }^{3} \mathrm{He}$ are participating. As a consequence, the orbital momentum wave function of the pair of ${ }^{3} \mathrm{He}$ atoms corresponds to $p$-wave and the spin of the pair is equal to 1. But all other considerations of Pomeranchuk, particularly about entropy behavior in solid and liquid phases, remain valid also in this case. The only difference is that there are now 3 superfluid phases [27]. 


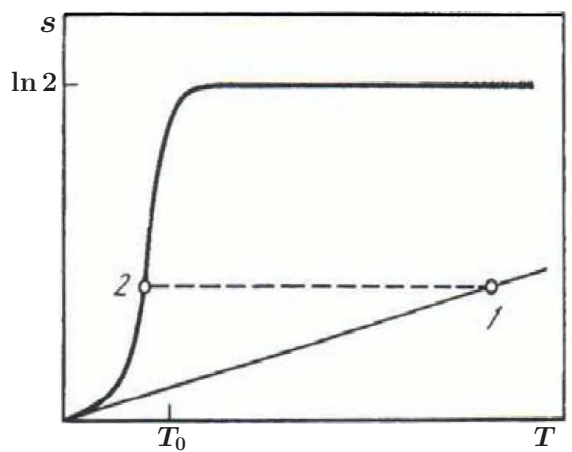

Fig. 1. The entropy dependence on temperature. Thick solid line and dashed line corresponds to entropy dependence on $T$ in solid and liquid phases at adiabatic cooling along the melting curve. The thin solid line corresponds to eq.(25).

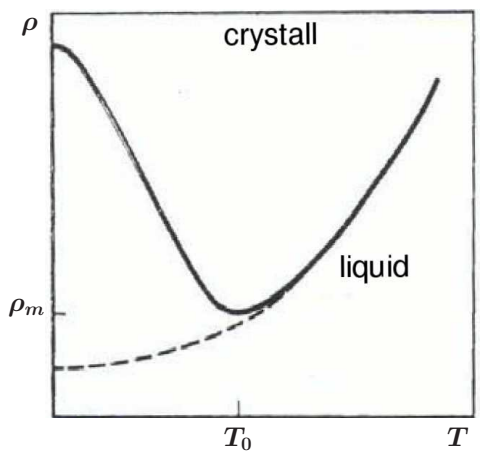

Fig. 2. The dependence of pressure on temperature at phase transition.

Using the Pomeranchuk method of cooling Osheroff, Richardson and Lee discovered the superfluidity of ${ }^{3} \mathrm{He}$. (Nobel prize, 1996) [28]. They found, that below $3 m K$ the liquid ${ }^{3} \mathrm{He}$ had 3 superfluid phases. All three are magnetic and have anisotropic behavior.

In paper [25] Pomeranchuk found also the temperature dependences of the specific heat of ${ }^{3} \mathrm{He}$, its viscosity and heat conduction. These results were confirmed later by the Landau theory of Fermi liquids and experiment.

Besides the study of ${ }^{3} \mathrm{He}$, Pomeranchuk (partly together with Landau) investigated the influence of small admixtures on the properties of liquid helium [29],[30].

\section{Quantum electrodynamics and quantum field theo- ries}

In ITEP we started to study the new approach to quantum electrodynamics - the Feynman, Schwinger, Dyson approach - in 1950. But before this, using the old technique - the Dirac Heitler formulation of QED - several important results were obtained. First, there was the fundamental theorem, proved by Berestetsky, that the parity of the positron is opposite to the parity of the electron [31]. Now this statement is trivial, you can find it in any textbook on QED. But because of its triviality, as a rule there are no references to the original Berestetsky paper, where the proof was presented for the first time, and few physicists in the West know by whom and where this theorem has been formulated. Berestetsky and Pomeranchuk calculated the cross section of $e^{+} e^{-}$annihilation into a $\mu^{+} \mu^{-}$pair [32]. Now this cross section is the standard theoretical scale and the cross sections of various $e^{+} e^{-} \rightarrow X$ processes are usually normalized to it. Pomeranchuk formulated the selection rules for $e^{+} e^{-}$ annihilation into photons [33]. He demonstrated, that the $e^{+} e^{-}$system with total spin $S=1$ cannot annihilate into two photons, and only annihilation into three photons is allowed for such a system. That means that the orthopositronium lifetime is much greater (by a factor of 700) than the lifetime of parapositronium, where two photon annihilation is allowed. These selection rules were generalized later by the famous Landau-Yang theorem, which states that two photons with opposite momenta cannot have a total angular momentum equal to 1. Also mentioned should be the formulation of the electron-positron interaction up to terms 
of order to $v^{2} / c^{2}$ with account of exchange interaction (Berestetsky, Landau [34]).

At the beginning of the 50-s only few people in Moscow studied the new developments in quantum field theory: the renormalization of masses and charges, the Feynman diagram technique etc. Landau was sceptical about them. Pomeranchuk tried to persuade him: "Dau, - he repeated, - there are many important and hard problems, which are just for you". (Dau, Chuk - the short names, which were used very often in Landau circles.) But Landau declined Chuk's suggestions: "To solve the problem of infinities is out of my possibilities" he replied. Two attempts to present talks about Feynman's work at the Landau seminar were unsuccessful: the speakers were thrown out in the middle of the seminar. Only the third time the speaker succeeded in finishing his talk, but also not with full success. Landau preferred to listen about his beloved subject - the alum . Landau called me "snob". Many times he repeated: "Boris is a snob". The meaning of his words was that I did not want to solve real physical problems, but preferred to study refined physical theories. His words did not influence Pomeranchuk, to whom they were usually addressed, since we had common views with Pomeranchuk. But, what was the worst, Landau said the same words to Alikhanov, the ITEP Director. For Alikhanov Landau was the unquestionable authority in theoretical physics. Therefore Landau's words could result in undesirable consequences for me. But luckily Alikhanov had his own opinion in this case. He knew well that I was performing calculations of nuclear reactors and of his, Alikhanov's, experimental devices, and that I was no snob by any means. Since Landau did not allow presentations of new developments in quantum field theory at his seminar, Pomeranchuk organized his own seminar devoted to quantum field theory. I was the secretary of this seminar and at the first meeting presented the Dyson paper.

In ITEP we were more interested in meson theories. At that time the only meson found experimentally was the pion, and it was known that the pion-nucleon coupling constant was large and perturbation theory was not applicable to the $\pi N$ interaction. (This statement was demonstrated in [35] by comparison with experiment of pseudoscalar meson theory with pseudoscalar and pseudovector couplings.) The idea was to write an infinite set of coupled equations for two-, three-, four- ... etc -point Green functions and perform in this system the mass and charge renormalization. After renormalization there should be no infinities in the equations. Then to cut the system: to consider, say, only the first two Green functions and find the solution neglecting the third, after that to take account of the third neglecting the fourth and so on. We hoped that such process would converge. I succeeded in writing such an infinite system of coupled equations for Green functions using the Schwinger functional equation [36] and expanding the Green functions, depending on an external source, as a functional series in this external source [37. But the equations so obtained were not suitable for mass and charge renormalization. Then Galanin, Pomeranchuk and myself [38] used another form of the Schwinger functional equations and obtained another infinite set of coupled equations for many-point Green functions. In this set it was possible to perform mass and charge renormalizations. However, it was impossible to cut the system: the factor $Z_{1}$, corresponding to renormalization of vertex functions appeared explicitly in the equations and in order to calculate it it was necessary to account for the Green functions beyond the cut. Otherwise the infinities did not cancel. So, this method had to be abandoned or modified.

In QED Galanin and myself had calculated the first two perturbative terms for the photon and electron polarization operators and for the vertex function. We found the appearance of terms $\sim e^{2} \ln \left(\left|p^{2}\right| / m^{2}\right)$ in the first order in $e^{2}$, where $p$ are the external momenta which we supposed to be of the same order and $p^{2}<0$. In the second order appeared 


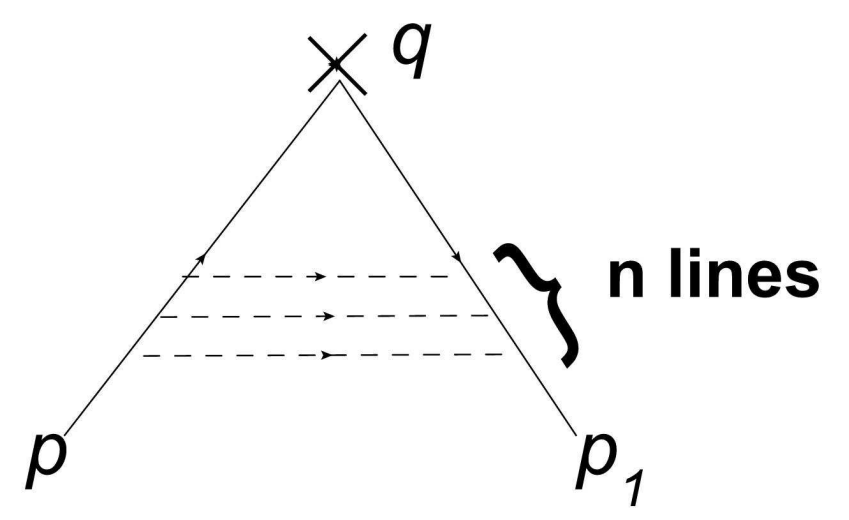

Fig. 3. Ladder approximation for $\bar{e} e \gamma$ vertex function. Solid and dashed lines correspond to electron and photon propagators. It is supposed, that $\left|p^{2}\right| \sim\left|p_{1}^{2}\right| \sim\left|q^{2}\right|$. The sum over $n$ should be performed.

terms $\sim\left(e^{2} \ln \left|p^{2}\right| / m^{2}\right)^{2}$. Very instructive for us was the paper by Edwards [39], who constructed a ladder equation for the vertex function and found that in the $n$-th order of perturbation theory terms of order $\left(e^{2} \ln \left|p^{2}\right| / m^{2}\right)^{n}$ persist. Evidently, these terms are the most important at large $\left|p^{2}\right|$.

In the 50-s Landau was coming to ITEP every week on Wednesdays, visiting Alikhanov's experimental seminar. After the seminar he went to the theory room, engaging in discussions for 1-2 hours. In one such discussion Galanin and I told him about our calculations and Edwards results. Next Wednesday Landau showed us his new idea: the point-like interaction in QED should be changed by a smeared one, characterized by some radius $a$ (or, in momentum space, by the cut-off $\Lambda$ ). In the calculation with smeared interaction all the most important terms of order $\left(e^{2} \ln \left|p^{2}\right| / \Lambda^{2}\right)^{n}$ must be accounted for. In Landau's approach the equation for the vertex function corresponds to the diagram of Fig.4. The equations for the exact electron and photon propagators $G$ and $D$ coincide with the ones proposed by Schwinger and Dyson. Landau expected that after realization of this program, i.e. after determination of $G, D$, and $\Gamma$, it would be possible to go to the limit $\Lambda \rightarrow \infty$ and all infinities in the theory would disappear. So, he expected that the theory would be asymptotically free. (This is even more than an asymptotically free theory, since in asymptotically free theories the infinities do not disappear: they can only be safely renormalized.) This Landau expectation can be easily noticed by looking at the title of the first Landau, Abrikosov and Khalatnikov paper: "On the removal of infinities in quantum electrodynamics". The Landau et al. papers were published as 4 papers in Doklady [40]. The first paper was published before the whole calculation was finished. Landau did not possess the Feynman technique, therefore all calculations were done by Abrikosov and Khalatnikov. But the basic ideas of the calculations were formulated by Landau: the choice of gauge, the rotation of the integration contour to the imaginary axis etc.

Here is the suitable place to say few words about Landau. Landau was an extraordinary physicist: he knew well the whole of theoretical physics. Not one such physicist existed in the past and will not exist in future: physics has become too extensive now. There is a saying attributed to Einstein: God performs integrations in the mind. Landau was not like that God, but his ability to do calculations was striking. One example. At the beginning of the 50-s Pomeranchuk gave me a problem to solve. I worked about a week and solved the problem by two methods which, I believed, were equivalent. But the results were different. I checked the calculations, but found no errors. I told Pomeranchuk about this discrepancy, 


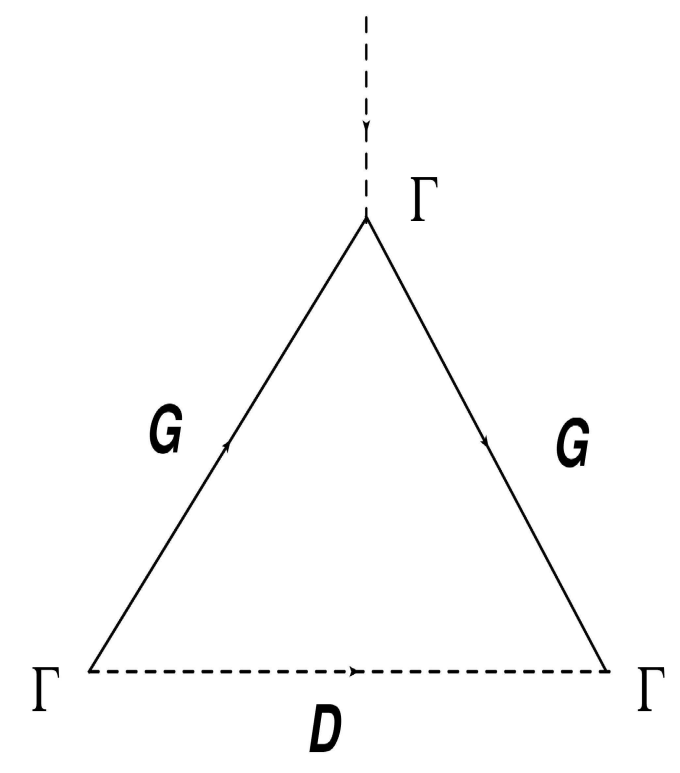

Fig. 4. Landau equation for vertex function $\Gamma$. $G$ and $D-$ are exact electron and photon propagators.

and in a few days he performed his own calculations. His results coincided with mine: the discrepancy still persisted. When Landau came to ITEP the next time, Pomeranchuk told him about this problem. Landau took a stick of chalk and started to write formulas at the top of the blackboard. After about 20 minutes the result appeared in the lower part of the blackboard: it coincided with one of ours. But from the derivation it was clear that this result was correct and the other one was wrong.

The main result of Landau, Abrikosov and Khalatnikov's calculations was presented in terms of an effective electron charge $e^{2}(\Lambda)$, defined at the scale of cut-off $\Lambda$. One Wednesday Landau presented to us the result of their calculations:

$$
e^{2}(\Lambda)=\frac{e^{2}}{1+\frac{e^{2}}{3 \pi} \ln \frac{\Lambda^{2}}{m^{2}}},
$$

where $e^{2}$ is the physical charge. The behavior of $e^{2}(\Lambda)$ agreed with Landau's expectations: $e^{2}(\Lambda)$ tends to zero, when $\Lambda$ tends to infinity. Galanin and myself decided to check this result. It was easy to do it, because Landau, Abrikosov and Khalatnikov had demonstrated that in Landau gauge, when the photon propagator is transverse, the electron polarization operator and vertex function do not give terms $\sim e^{2} \ln \left(\left|p^{2}\right| \Lambda^{2}\right)$, and such terms arise only from the photon polarization operator. We calculated it and found that in eq.(27) there was a mistake of the sign in the denominator. The correct equation should be

$$
e^{2}(\Lambda)=\frac{e^{2}}{1-\frac{e^{2}}{3 \pi} \ln \frac{\Lambda^{2}}{m^{2}}}
$$


The effective electron charge increases with momentum and tends to infinity when $\left(e^{2} / 3 \pi\right) \ln \left(\Lambda^{2} / m^{2}\right)=1$. The correct eq.(28), expressing the physical charge in terms of the bare charge $e^{2}(\Lambda)$ defined at the cut-off, looks like

$$
e^{2}=\frac{e^{2}(\Lambda)}{1+\frac{e^{2}(\Lambda)}{3 \pi} \ln \frac{\Lambda^{2}}{m^{2}}}
$$

So, at arbitrary $e^{2}(\Lambda)$, when $\Lambda^{2} \rightarrow \infty$, the physical charge is going to zero. The theory became contradictory and the problem is known now as the problem of vanishing of the physical charge. On the following Wednesday we told Landau about this error. He checked our calculation and agreed with us. As told by S.Gershtein, who was working at the Landau Theory Division in Kapitza's Institute, Landau came back from ITEP with the words: "Galanin and Ioffe saved me from shame!" (Later Gershtein published this story in his recollections.)

The physical interpretation of eq.(28) is the folloing: Landau equations, defined by contributions of diagrams of Fig.4, are valid at photon and electron virtualities $p^{2}$, when

$$
1-\frac{e^{2}}{3 \pi} \ln \frac{\left|p^{2}\right|}{m^{2}} \lesssim e^{2}
$$

At higher $\left|p^{2}\right|$ the contributions of diagrams, not represented in the form of Fig.4, become important. A similar situation arises in Yukawa meson theories as was demonstrated in [41, [42]. (The latter paper was done within the framework of the approach [38], when the renormalization was performed $a b$ initio, before the calculation.)

Landau and Pomeranchuk demonstrated, that the trouble arising is not only the defect of Landau equations, but is much more general [43]. Their arguments were the followings. The term 1 in the denominator in eq.(29) corresponds to the free term contribution in the Lagrangian. If this term gives a small contribution in (29) at large $\left(e^{2}(\Lambda) / 3 \pi\right) \ln \left(\Lambda^{2} / m^{2}\right)$ and can be neglected, then there are even stronger reasons to neglect this term, when the interaction in QED becomes strong, i.e. the second term in the denominator is very large. In such case we came to the conclusion that quantum electrodynamics is a contradictory theory. The same conclusion holds for meson theories. In QED the contradiction is nonessential practically, because it arises at energies unreachable by experiment. In meson theories, since the coupling constant $g^{2} / 4 \pi$ is large, the contradiction appears at energies of the order of 1 $\mathrm{GeV}$. Therefore, such theories must be rejected.

As is well known now, this problem was solved by the discovery of nonabelian gauge theories, which have the property of asymptotic freedom.

An important invention in QED was done by Sudakov [44]. He found that in the electron form factor at high momentum transfer $q=p_{2}-p_{1}$, there appear terms proportional to the square of $\ln q^{2}$, like $\left(e^{2} \ln ^{2} q^{2} / m^{2}\right)^{n}$ in $n$-th order of perturbation theory, and succeeded in summing all of them. Such terms arise from diagrams of which an example is shown in Fig.5. The photon lines connect the initial and final electron propagators. Their number and their order on both electron lines is arbitrary and the sum must be performed over all such diagrams and over powers $n$ in perturbation theory. Other diagrams where the photon line connects the same electron line or diagrams with electron loops do not result in the appearance of $\left(e^{2} \ln ^{2} q^{2} / p^{2}\right)^{n}$ terms. It is supposed that $\left|q^{2}\right| \gg p_{1}^{2}, p_{2}^{2}$, i.e. the terms proportional to $\left(e^{2} \ln q^{2} / p^{2}\right)^{n}$ are neglected. Such approximation is called double logarithmic approximation. If $p_{1}$ and $p_{2}$ are on the mass shell, $p_{1}^{2}=p_{2}^{2}=m^{2}$, then the contribution of double logarithmic terms corresponds (at $q^{2}<0$ ) to the electron electromagnetic form 


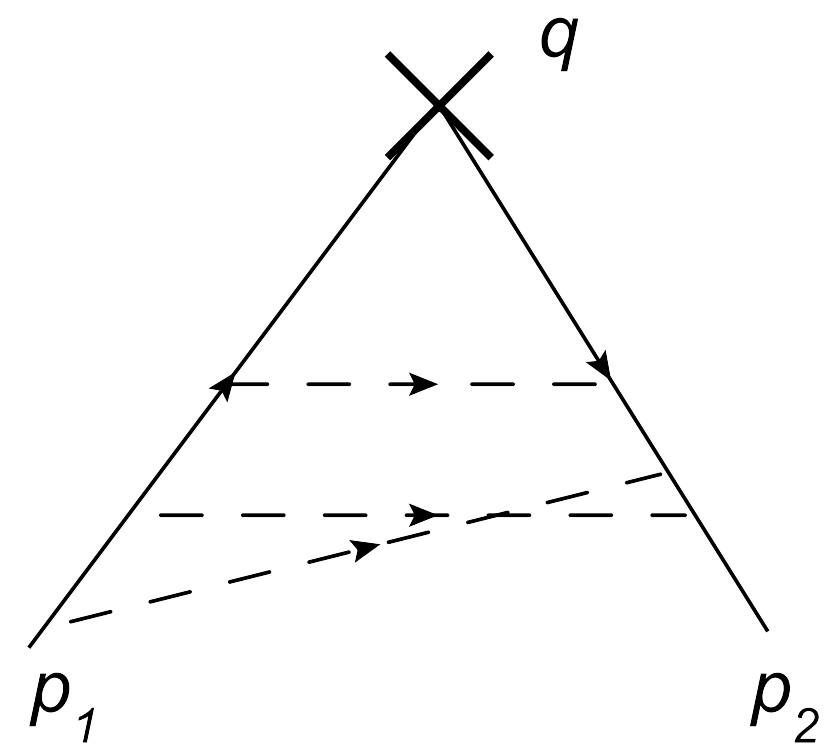

Fig. 5. Example of a diagram where the $\ln ^{2}\left(q^{2} / m^{2}\right)$ term appear.

factor, and to the electron vertex function $\Gamma\left(p_{1}^{2}, p_{2}^{2} ; q^{2}\right)$ when $p_{1}^{2}$ and $p_{2}^{2}$ are off mass shell. The appearance of double logarithmic terms can be most easily seen by using the Sudakov decomposition of the photon momentum $k$ of any photon line in diagrams of the type of Fig.5:

$$
k=u\left(p_{1}-\alpha_{1} p_{2}\right)+v\left(p_{2}-\alpha_{1} p_{1}\right)+k_{\perp},
$$

where $k_{\perp}$ is the 4 -vector orthogonal to $p_{1}$ and $p_{2}$ :

$$
k_{\perp} p_{1}=k_{\perp} p_{2}=0 \text {. }
$$

The conditions

$$
\left(p_{1}-\alpha_{1} p_{2}\right)^{2}=\left(p_{2}-\alpha_{2} p_{1}\right)^{2}=0
$$

are imposed on the parameters $\alpha_{1}$ and $\alpha_{2}$, which defines them unambiguously. By considering the simplest diagrams of the type of Fig.5 - the diagram of Fig.4, where $G$ and $D$ are free propagators and $\Gamma_{\mu}=\gamma_{\mu}$, - it can be shown that the integration over $k_{\perp}$ does not result in the appearance of logarithmic terms. The double logarithmic terms arise from the integration over $u$ and $v$ and they are proportional to $\ln \left(q^{2} / p_{2}^{2}\right)$ and $\ln \left(q^{2} / p_{1}^{2}\right)$, respectively. The consideration of more complicated diagrams can be performed in a similar way. In $n$-th order of perturbation theory the contributions of diagrams, which are different from one another by transposition of initial or final points of photon lines are equal. The final result is:

$$
\Gamma_{\mu}\left(p_{1}, p_{2} ; q\right)=\gamma_{\mu} \exp \left[-\frac{\alpha}{2 \pi} \ln \left(\frac{q^{2}}{p_{1}^{2}}\right) \ln \left(\frac{q^{2}}{p_{2}^{2}}\right)\right], \quad \alpha=e^{2}=1 / 137
$$

at $q^{2}<0, p_{1}^{2}<0, p_{2}^{2}<0$. The result is valid if

$$
\ln \frac{q^{2}}{p_{1}^{2}} \gg 1, \quad \alpha \ln \frac{q^{2}}{p_{1}^{2}} \ll 1, \quad \alpha \ln ^{2} \frac{q^{2}}{p_{1}^{2}} \lesssim 1
$$

In case of electrons on mass shell (electron form factor) $p_{1}^{2}$ and $p_{2}^{2}$ must be replaced by $m^{2}$ and $q^{2}$ by $\left|q^{2}\right|$.

A similar technique was applied to calculate the double logarithmic asymptotics in many other processes. But some specifics may arise. For example, in case of high energy electron 
elastic scattering in external electromagnetic field, where the emission of soft photons by the electron must be accounted for, the corresponding formula for the cross section is [45], [46]

$$
d \sigma=d \sigma_{0} \exp \left[-\frac{2 \alpha}{\pi} \ln \frac{\left|q^{2}\right|}{m^{2}} \ln \frac{E}{\Delta E}\right]
$$

where $\sigma_{0}$ is the cross section in Born approximation, $E$ is the electron energy and $\Delta E$ is the experimental resolution, inside which the soft photons are not detected (or the uncertainty in the measurement of electron final energy). Double logarithmic terms play an important role in the production of narrow resonances $V$ in $e^{+} e^{-}$annihilation,

$$
e^{+} e^{-} \rightarrow V
$$

where the resonance can be produced not only when the $e^{+} e^{-}$energy is equal to $m_{V}$ (in this case the double logarithmic terms must be accounted for), but at a slightly higher energy, when the $e^{+}$and $e^{-}$are emitting soft photons before annihilation (see [47] and references therein).

Last not least must be mentioned the book "Quantum Electrodynamics" written by A.Akhiezer and V.B.Berestetsky [48]. The first edition of the book was published in 1953 in Russian. Later the content of the book was extended and improved and new editions of the book were published. The book was translated into English. Now in my opinion it is the best book on QED. I recommend to students as well as to qualified specialists to read it. (Of course, the parts of interest to them : to study the whole book is a very hard task.) V.Berestetsky, a member of the ITEP Theory Group (in 1966-1977 the Head of the ITEP Theory Department) was also a co-author of one volume of the famous Landau-Lifshitz Course of Theoretical Physics [49].

\section{Parity violation in weak interactions. The theory of $\beta$-decay and other weak processes}

In 1955-56 the $\theta-\tau$ puzzle agitated all physicists. The $K$-meson decays in 2 and 3 pions had been observed experimentally. Under the condition of parity conservation, which was taken for granted at that time, one and the same kaon could not decay sometimes into 2 and sometimes into 3 pions. For this reason most physicists believed that $\theta$, decaying into 2 pions and $\tau$, decaying into 3 pions, were different mesons. As the precision of experiments grew, it became clear, however, that their masses coincided. At that time, in spring of 1956, Lee and Yang came up with their revolutionary paper [50], in which they proposed the hypothesis of nonconservation of parity $(P)$ in weak interactions, that explained the $\theta-\tau$ puzzle. Moreover, Lee and Yang calculated parity nonconservation effects in $\beta$ decay and in $\pi \rightarrow \mu \rightarrow e$ cascade decay.

Landau vigorously rejected the possibility of parity nonconservation, saying: "space cannot be asymmetric". Pomeranchuk was attracted more by a hypothesis of parity-degenerate doublets of strange particles. A. Rudik and I decided to calculate some additional effects based on the assumption of parity nonconservation in weak interactions, other than those considered by Lee and Yang. Our choice was the $\beta-\gamma$ correlation. I made an estimate and found that the corresponding effect had to be large. Rudic turned to a detailed calculation. After some time he told me:

"Look, Boris, the effect vanishes." 
"This cannot to be case", I replied.

We started to examine the calculation and I noticed that Rudik, being a well-educated theorist, had imposed the condition of charge $(C)$ invariance on the weak interaction Lagrangian. As a result the coupling constants multiplying the parity non-conserving terms turned out to be pure imaginary. The constants in the Lee and Yang paper were arbitrary complex numbers. (If one assumes them to be pure imaginary, then all parity non-conserving effects disappear.)

The question arose about the connection between $C$ and $P$ invariance. In a discussion of this problem with Volodya Sudakov we remembered a paper by Pauli [51] related to this subject and published about a year ago. Although I had read this paper previously, I had forgotten about it. In part the reason was that Landau regarded this paper with scepticism (although he had a great respect for Pauli): he believed that the $C P T=1$ theorem $(T$ means the time inversion) was a trivial relation satisfied by any Lagrangian, and no physical consequences could follow from the CPT theorem. I noted that Lee and Yang in their paper did not mention the $C P T$ theorem at all, and nothing was said on the connection between $C, P$ and $T$ invariance. I read the Pauli paper again, with more attention than at first, and it became clear to me that if $P$ is violated, then $C$ or $T$ or both had to be violated with certainty.

Then came the following idea. The existence of two $K^{0}$-mesons with strongly different lifetimes had been proved by Gell-Mann and Pais by assuming $C$-invariance of the weak interaction Lagrangian. A similar proof can be made if $T$-invariance is applied. If $C$ and $T$-invariance are both violated to comparable degrees, then $K_{S}$ and $K_{L}$ would have similar lifetimes. Experimentally, their lifetimes differ by two orders of magnitude. The conclusion is, that one of two conservation laws, $C$ or $T$ conservation, had to be valid, at least approximately. Rudik and I considered a number of effects and found, that $P$-odd pair correlations of spin and momentum (the terms $\boldsymbol{\sigma} \mathbf{p}$ ) appear if $C$ is violated and $T$ is conserved. In the opposite case they are absent. (In my subsequent paper [52] - paper $\mathrm{V}$ in the Appendix - I proved this theorem in a general form and found the type of $P$-odd terms corresponding to $T$-violation.) We wrote a paper and I told L.Okun about its content. Okun made the very useful remark that similar effects, which unambiguously differentiate the $C$ invariant theories from $T$-invariant ones, appear in $K^{0}$ decays too. We included this remark in the paper and I suggested to Okun to become a co-author. At first he refused, saying that such a remark deserves only to be mentioned in the acknowledgement, but later I persuaded him. After that I reported our results to Pomeranchuk. Pomeranchuk decided that we had to make our results known to Dau - immediately, next Wednesday. On Wednesday Dau's first reaction was to refuse to listen: "I do not want to hear anything about parity nonconservation. This is nonsense!"

Chuk persuaded him: "Dau, have patience for 15 minutes, listen to what the young people have to say."

With heavy heart Dau agreed. I spoke not for long, perhaps for half an hour. Dau kept silent, and then went away. Next day in the morning Pomeranchuk called me: Dau had solved the parity nonconservation problem ! We were supposed to come to him immediately. By that time both of Landau's papers - on the conservation of combined parity ( $C P$-conservation) and on the two-component neutrino were ready with all formulations. In the first paper he gave an acknowledgement to us: "I would like to express the deep gratitude to B.Ioffe, L.Okun and A.Rudik, in a discussion with whom the idea of this work has arisen."

Our paper [53] and those of Landau [54, [55] were sent for publication prior to the paper by $\mathrm{Wu}$ et al., [56], where the observation of electron asymmetry in the decay of polarized 
nuclei was reported (i.e. the correlation between nuclear spin and electron momentum). In this way the parity nonconservation was discovered. Our results implied that $C$-parity was not conserved in $\beta$-decay either. The corresponding statement was added in proof to our paper. An similar statement was made in the paper by $\mathrm{Wu}$ et al [56], who referred to the paper by Lee, Oehme and Yang [57], which in turn was published after our paper. In their Nobel lectures Lee and Yang emphasized our priority in this problem. Later for this work we got the USSR Prize of Discovery.

Unfortunately, the story of the creation of these Landau works finished with an unpleasant episode, which I shall mention for objectivity reasons. A few days after the presentation of his papers to ZhETF Landau gave an interview to a correspondent of "Pravda", the most important newspaper in the USSR. In this interview Landau told about the problem of parity nonconservation and explained how he had solved it. The work of Lee and Yang was not referred to (not to mention our paper). All ITEP theoreticians were indignant by this interview. Berestetsky and Ter-Martirosyan visited Landau and told him, what they thought about this interview. The result followed immediately: both of them were excommunicated from the Landau seminar. I did not give a piece of my mind directly to Landau but presented it to his collaborators, who perhaps passed it on to Landau. I was punished in another way: Landau cancelled my name in the acknowledgement in his paper, leaving only Okun and Rudik. Here Pomeranchuk was not be able to stick it any longer. He visited Landau and said to him (this Chuk told me later): "Boris explained to you everything about $C, P$ and $T$. Without him your work would not have been done, and you cancel his name in the acknowledgement !" Landau made a compromise: he restored my name in the acknowledgement, but not in alphabetical order - only as the second name. Landau considered $C P$ conservation to be an exact law of the nature: he did not admit its violation. Concerning $C P$ Landau would say exactly the same words on the space asymmetry as he used to say previously with regards to $P$ violation. I constructed an example of a Lagrangian in which $C P$ was violated and nothing happened to the vacuum and tried to change Landau's mind, but he did not want to listen. The $C P$ violation was discovered by Christenson, Cronin, Fitch and Turley [58] in 1964, when Landau was in very bad health, damaged in a car accident.

In a paper by Berestetsky et al. [59] the theory of $\beta$-decay was developed on the most general grounds: all possible 5 variants of $\beta$-interaction with parity violating terms were accounted for as well as the effects of Coulomb field and finite nuclear size. $C P$-conservation was not assumed. Results were obtained for allowed transitions, first order forbidden transitions in intermediate and heavy nuclei (Coulomb transitions), unique transitions [ $\Delta j=2$, (yes)] and 0-0 (yes) transition. Angular distributions of electrons and neutrinos (equivalent to recoil nuclei distributions) were calculated for unpolarized and polarized initial nuclei. The degree of polarization of $\beta$-decay electrons was also determined. Particulary, it was shown that $C P$-violation appears in the distribution proportional to the term $\mathbf{n}_{j}\left[\mathbf{n}_{e}, \mathbf{n}_{\nu}\right]$, where $\mathbf{n}_{j}$ is the direction of nuclear spin, $\mathbf{n}_{e}$ and $\mathbf{n}_{\nu}$ are the directions of the electron and neutrino momenta.

Okun and Pontecorvo demonstrated [60], that if a weak interaction with change of strangeness by 2 units and with a coupling constant of the order of the Fermi coupling constant exists, $G(\Delta S=2) \sim G_{F}$, then the mass difference $m_{K_{1}}-m_{K_{2}}$ would be greater than its actual value by many orders of magnitude. Therefore it was proved that $G(\Delta S=2) \ll G_{F}$ and, probably, of the order of $G_{F}^{2} m^{2}$.

In 1958 Feynman and Gell-Mann [61] and Marshak and Sudarshan [62] proposed a universal form of $V-A$ weak interaction. According to [61] the vector current that enters the 
weak interaction belongs to the same isotopic triplet as the vector part of electromagnetic current. (Earlier the same hypothesis was formulated by Gershtein and Zeldovich [63]). This hypothesis was called the hypothesis of conserved vector current $-C V C$. As a check of the $C V C$ hypothesis Gell-Mann [64] proposed the measurement of the "weak magnetism" - the correction term to the neutron-proton weak vector form factor, which was calculated unambiguously in [64] within the framework of $C V C$. Another method to check the $C V C$ hypothesis was suggested by Vaks and Ioffe [65]. Basing on the $C V C$ theory they found the ratio of the $\pi \rightarrow e \nu \gamma$ decay width through the vector current to the $\pi^{0} \rightarrow 2 \gamma$ decay width and predicted the electron spectrum in $\pi \rightarrow e \nu \gamma$ decay through vector current. Both checks were confirmed by experiment and now $C V C$ is one of the corner stones of the Standard Model of weak interaction.

\section{Strong interactions and nuclear physics}

In the 1950-s K.Ter-Martirosyan produced two very good papers on nuclear physics. In the first of them [66] he calculated the excitation of nuclei by the electric Coulomb field of a charged particle passing near the nucleus. In the second [67] he and Skornyakov solved the three-body problem in quantum mechanics for the case of an infinitely small radius of two-body interaction. The solution of this problem has direct applications to the case of interaction of three nuclei at low energies, their scattering, formation of bound states etc. In [67] the equations for the three-body problem were presented in differential form. Later they were reformulated by Faddeev in integral form, which is more convenient for analysis. Till now these equations are widely used in many branches of physics.

Great attention was paid to diffraction production of particles in $p-p$ and $p$-nucleus collisions at high energies (see e.g. [68]-[70]). The main idea was that the minimal momentum transfer from proton to nucleus in, say, pion production in $p A$ collisions is equal to $q=m \mu / E$, where $m$ and $\mu$ are the proton and pion masses, and $E$ is the proton energy. If $1 / q \gg R$ - the nuclear radius, - then the pion production process proceeds outside the nucleus and the characteristics of such a process can be calculated phenomenologically without the use of perturbation theory.

By this method a number of processes were calculated: elastic diffraction scattering in $p p$ and $p A$ collisions, production of photons, mesons and meson pairs in $p A$ collisions, diffraction phenomena in deuteron-nucleus scattering, photon production in collisions of mesons with nuclei etc. When Pomeranchuk reported the results of these calculation at a seminar of the Lebedev Institute, Academician Skobelzyn asked: "How can it be that the production process proceeds outside the nucleus?" Pomeranchuk explained that the wave function of the incoming particles overlaps with the shadow of the nucleus, which results in a distortion of the wave function and gives rise to the production processes. Then he continued his talk. After some time Skobelzyn repeated his question. Pomeranchuk gave the same explanation, but in more detail. After another while Skobelzyn repeated his question for a third time. Pomeranchuk's reply was: "If you like, you can consider this effect as immaculate conception".

Pomeranchuk developed the statistical approach to multiparticle production in high energy collisions, suggested by Fermi [71]. Fermi supposed that in high energy collisions the whole energy of the colliding particles (e.g. nuclei) is concentrated in the small Lorentz contracted volume

$$
\Omega=\Omega_{0} \frac{2 M c^{2}}{W}=\frac{4 \pi}{3}\left(\frac{\hbar}{\mu c}\right)^{3} \frac{2 M c^{2}}{W},
$$


where $M$ and $\mu$ are the nuclear and pion mass, respectively, and $W$ is the total energy in the c.m. system. In this volume a statistical equilibrium is set up and the probabilities of various states are proportional to their statistical weights. Pomeranchuk remarked [72] that in such a small volume the produced particles are strongly interacting with one another, which results in the production of new particles and expansion of the fireball. This process will continue till the distances between particles will be of the order of $\hbar / \mu c$. It follows from Pomeranchuk's statement that the mean multiplicity $\bar{N}$ of produced particles at high energies is proportional to $W$ :

$$
\bar{N} \approx \frac{W}{\varepsilon},
$$

where $\varepsilon$ is of the order of a few hundred MeV, in comparison with Fermi's prediction $\tilde{N} \sim$ $\sqrt{W}$. The Pomeranchuk picture of multiparticle production was exploited by Landau and Belenky [73] in their hydrodynamical model of high energy collisions and the latter is widely used now in the description of heavy ion collisions.

When the charged pions had been discovered but not yet the $\pi^{0}$, the physical community was divided in two groups: one of them believed that the $\pi^{0}$ exists and will be found, the other denied its existence. Landau did not believe that the $\pi^{0}$ could exist and often repeated: "God will not permit the existence of a diphoton". Later, when the $\pi^{0}$ was discovered, people who opposed his statements said in discussions with him: "But Dau, God did permit the existence of the diphoton".

After the discovery of the $\pi^{+}, \pi^{-}$and $\pi^{0}$ several papers at ITEP were the devoted to determination of their spin and parity. The spin 0 of $\pi^{0}$ follows directly from its decay into two $\gamma^{\prime}$ s. It was natural to assume, that the $\pi^{ \pm}$spin is also equal to 0 . The negative parity of $\pi^{-}$(and $\pi^{+}$) follows from the observation of the reaction

$$
\pi^{-}+d \rightarrow n+n
$$

for slow $\pi^{-}$(captured from the $S$-level) [74]. In order to determine the parity of $\pi^{0}$ calculations of the following processes were performed [75]:

$$
\begin{gathered}
\pi^{-}+d \rightarrow n+n+\gamma, \\
\pi^{-}+d \rightarrow n+n+\pi^{0},
\end{gathered}
$$

when $\pi^{-}$is captured from the $S$-state in deuterium. The calculations were done in perturbation theory. (From the modern point of view this is the correct approach, because the pion-nucleon interaction tends to zero at small momenta.) Two theoretical tricks were used in these calculations: 1) the nonrelativistic expansion of the Hamiltonian in the operator form; 2) the use of the completeness theorem for the determination of the total probability in case of the process (41). Strong suppression of reaction (42) in comparison with (41) was found in case of negative parity of $\pi^{0}$. Just such suppression was found experimentally. The conclusion was that the spin-parity of $\pi^{ \pm, 0}$ is $J^{p}=0^{-}$. Later Pomeranchuk demonstrated that these processes can be calculated phenomenologically, without the use of perturbation theory, leading to the same conclusion [76].

Pomeranchuk had shown that at high energies the annihilation cross sections of antiprotons with proton and neutron are equal [77], Okun and Pomeranchuk demonstrated the equality at high energies of the total cross sections of $\pi^{+}, \pi^{-}$and $\pi^{0}$ on proton [78]. Basing on the statement that the scattering amplitudes at high angular momenta are determined by the exchange of the lightest particle - the pion, - Okun and Pomeranchuk formulated the calculation method to determine this amplitude [79]. 
The peak of the investigations of strong interactions in ITEP at the period under consideration was the famous Pomeranchuk theorem [80]. This theorem states that asymptotically at high energies the total cross sections of protons and antiprotons on any target are equal. (The same statement holds for $n$ and $\bar{n}$ [80]). In the proof of this theorem Pomeranshuk exploited the dispersion relations for the amplitudes of proton and antiproton scattering formulated in [81, 82] and the assumptions that $\sigma(p), \sigma(\bar{p})$ and the radius of interaction tend to constant values at high energies. Although these assumptions are not correct according to the modern point of view, the Pomeranchuk theorem is probably valid.

Finally, I would like to mention the book by A.Akhiezer and I.Ya.Pomeranchuk "Some problems in theory of nucleous" [83. A lot of problems we considered in this book: proton -neutron interaction, statistical description of heavy nucleous, the resonance phenomena in the scattering of neutrons on nucleous, the neutron moderation in the medium, the fission of heavy nucleous, the propagation of neutrons in crystalls.

\section{The contacts of Russian physicists with their West- ern colleques in 1955-1957}

In the years 1945-1955 any contacts of Russian physicists with their Western colleques were strictly forbidden. The same prohibition refered also to publication of the papers in Western journal, written by Russian authors. In this connection an interesting episode happened. At the year 1946 Pomeranchuk wrote a paper about $\lambda$-limiting process in quantum field theory. (the $\lambda$-limiting process for removing the infinities in quantum field theories was suggested by Wentzel [84] and Dirac [85]). Pomeranchuk demonstrated that this method is not selfconsistent and the results of its application are not unique. The paper was send in Physical Review in 1946. At this time the Cold War only starts and such violation of the rule was not dangerous. For unknown to us reasons the paper was published only in 1949 [86], when the Cold war was in the full swing. Pomeranchuk was afraid, that he will be punished. But nothing happened, perhaps the censorship overlooked the appearance of this paper.

The contacts were renewed in 1955. The first Western physicist, who came to Moscow was G.Källen from Sweden. He presented a talk at Lebedev Institute. Since it was a very unusual event a lot of people came to listen his talk - about 400 persons. In his talk he presented the consideration of the Lee model at high energies done by him and Pauli. The result was qualitative similar to the result of Landau, Abrikosov and Khalatnikov - the theory is inconsistent at high energies - the unphysical pole appears in the expression for Green functions and effective charge. However, Lee model has many drawbacks: the crossing symmetry was absent, the causality principle was badly violated etc. These defects of the model were mentioned by the participants of the seminar.

Some contacts of Russian and Western physicists took place at the 1-st International Conference on the Peaceful Use of Atomic Energy, which took place at Geneve in 1955. The number of these contacts was restricted: the atmosphere at the Conference was very official. But in other aspect the Conference was very effective; many papers from both sides were declassified and presented at the Conference.

At 1956 very representative delegation of Western theoreticians came to Moscow. Among

the members of the delegation were: Dyson, Gell-Mann, Marshak, Pais, Weisskopf, Low, Brueckner. A Conference was organized at the Institute of Physical Problems (Kapitza Institute) at which from the Russian side were presented the talks: by Landau on the inconsistency of QED ("zero charge" problem); by Pomeranchuk about diffractional production 
and scattering; Bogoljubov presented the strict proof of dispersion relation for forward pionnucleon scattering amplitude etc. The Western participants also presented their talks. But the most important for us, the Russian participants, were the private conversation with leading Western scientists, in which we could understand the direction of future development of our science.

After the publication of Landau, Abrokosov and Khalatnikov's paper in approximately one year, Landau got a letter from Pauli. In this letter Pauli informed him that his graduate student, Walter Thirring, had found an example of the theory, in which there was no zero charge problem - the theory of scalar meson-nucleon interaction. The manuscript of Thirring's paper was attached to the letter. Landau gave this paper to Pomeranchuk and Pomeranchuk asked me to check the paper. I studied Thirring's paper and came to the conclusion that it was wrong. The origin of the mistake was that, in the paper it was exploited the Ward identity arising from differentiation over nucleon mass, which in fact was violated by renormalization. I told Pomeranchuk about this.

- "You should write a letter Pauli" - was Pomeranchuk responce.

I hesitated: to write to Pauli, that his graduate student had make a mistake and he, Pauli, had overlooked it ! Pomeranchuk insisted and, finally, I wrote a letter. The answer I received, was not from Pauli, but from Thirring. He accepted his error, and Thirring's paper never was published. Later we became good friends.

\section{Concluding remark}

Of course, only a part of the work done by ITEP theoreticians has been discussed in this review. The selection of the presented papers is not free from subjectivity. This is unavoidable. Only future historian, who can compare various points of view, will be able to be objective. But the witness of all described events has some advantage: he can present to the reader the atmosphere in which the events proceeded. This will be impossible for future historians.

I am very thankful to W.v.Schlippe, who improved the English and did valuable remarks. I am indebted to M.N.Markina for preparation of the manuscript.

This work was supported in part by CRDF Cooperative Program grant RUP2-2961-MO09 and RFBR grant 12-02-00284-a. 


\section{References}

[1] B.L.Ioffe, in: Handbook of QCD, ed. by M.Shifman, v.4, p.2528, World Sci., 2002.

[2] Ya.B.Zeldovich and Yu.B.Khariton, ZhETF, 10 (1940) 29.

[3] I.I.Gurevich and I.Ya.Pomeranchuk, 1st Intern. Conf. on the Peaceful Use of Atomic Energy, v.5 (1955) p.466.

[4] A.Rudik, Resonansnoe poglozhenie kolzevymi blokami c zamedlitelem vnutri [Resonance absorption by ring-shaped slabs with moderator inside], ITEP report No 280 (1950).

[5] A.I.Akhiezer and I.Ya.Pomeranchuk, Vvdedenie $\mathrm{v}$ teoriyu neitronnykh multiplitsiruyushchikh sistem [Introduction to the Theory of Neutron Multiplying Systems], IzdAT, Moscow, 2002.

[6] A.D.Galanin, Vvedenie v teoriyu yadernykh reaktorov na teplovykh neitronakh [Introduction to the Theory of Nuclear Reactors on Thermal Neutrons], Energoatomizdat, Moscow, 1990.

[7] A.D.Galanin, Teoria geterogennogo reaktora [The Theory of Heterogenous Reactor], Atomizdat Moscow, 1971.

[8] A.D.Galanin and B.L.Ioffe, Tyazhelovodny reaktor-brider, ispolsuychi cycl $U-T h$ [Heavy water reactor-breeder using $U-T h$ cycle], ITEP report No 319 (1950).

[9] B.L.Ioffe and L.B.Okun, Atomnaya Energia, 1, No. 4 (1956) 80.

[10] B.L.Ioffe, in: Problemy yadernoi fiziki I fiziki elementarnykh chastits [Problems of Nuclear and Elementary Particles Physics], Nauka, Moscow, 1975, 345.

[11] R.Rhodes, Dark Sun. The Making of Hydrogen Bomb, New York-London, Simon and Schuster, 1985.

[12] I.I.Gurevich, Ya.B.Zeldovich, I.Ya.Pomerancuk, Yu.B.Khariton, Physics-Uspekhi, 161 (1991) 171.

[13] G.A.Goncharov, Physics Today 49, November 1996, p.p.44-61.

[14] B.L.Ioffe, Bez retushi, [Without Retouching] (in Russian), Phasis, Moscow, 2004.

[15] B.L.Ioffe, Sechenie Komptonovskogo rasseynia na proizvolno dviszhuschemsya electrone [Cross section on arbitrary moving electron of compton scattering], ITEP report No. 370 (1952).

[16] B.L.Ioffe, ITEP report No. 779 (1953), Astronomy Letters, 20, (1994) No.6, 695.

[17] V.B.Berestetsky, B.L.Ioffe and I.Ya.Pomeranchuk, in: I.Pomeranchuk, Collected Works, v.1, p.345, Moscow Nauka, 1972 (in Russian, Appendix, paper III).

[18] L.D.Landau, Phys.Zs.Sowjet. 10 (1936) 154.

[19] I.Ya.Pomeranchuk, J.Phys. USSR 2 (1940) 65. 
[20] D.D.Ivanenko and I.Ya.Pomeranchuk, Doklady AN USSR, 44 (1944) 349 (Appendix, paper I).

[21] L.A.Arzimovich and I.Ya.Pomeranchuk, J.Phys.USSR, 9 (1945) 267.

[22] L.D.Landau and I,Ya.Pomeranhuk, Doklady AN USSR, 92 (1953) 535, 735.

[23] A.B.Migdal, Doklady AN USSR, 96, (1954) 49.

[24] A.I.Akhiezer and N.F.Shulga, Elektrodinamika vysokikh energii v veshchestve [Electrodynamics at high energies in matter] (In Russian), Nauka, Fizmatlit, Moscow, 1993.

[25] I.Ya.Pomeranchuk, ZhETP, 20 (1950) 919.

[26] A.F.Andreev, in: Recollections on I.Ya.Pomeranhuk, (in Russian), Nauka, Moscow, 1988, p.266.

[27] B.V.Mineev and K.V.Samokhin, Vvedenie v teoriyu neobychnykh sverkhprovodnikov [Introduction to the theory of unusual superconductors], Ed. by MFTI, 1998.

[28] D.D.Osherov, R.C.Richardson and D.M.Lee, Phys.Rev.Lett. 28 (1972) 885.

D.D.Osherov, W.J.Gully, R.C.Richardson and D.M.Lee, Phys.Rev.Lett. 29 (1972) 920.

[29] I.Ya.Pomeranchuk and L.D.Landau, Doklady AN USSR, 59 (1948) 669.

[30] I.Ya.Pomeranchuk, ZhETF, 19 (1949) 42.

[31] V.B.Berestetsky, ZhETF, 21 (1951) 93.

[32] V.B.Berestetsky and I.Ya.Pomeranchuk, ZhETF 29 (1955) 864.

[33] I.Ya.Pomeranchuk, Doklady AN USSR, 60 (1948) 213.

[34] V.B.Berestetsky and L.D.Landau, ZhETF, 19 (1949) 673.

[35] B.L.Ioffe and A.P.Rudik, ZhETF, 22 (1952) 128.

[36] J.Schwinger, Proc.Nat.Acad. Sci. 37 (1951) 452, 455.

[37] B.L.Ioffe, Doklady AN USSR, 45 (1954) 761.

[38] A.D.Galanin, B.L.Ioffe and I.Ya.Pomeranchuk, Doklady AN USSR 48 (1954) 361.

[39] S.F.Edwards, Phys.Rev.90 (1953) 284.

[40] L.D.Landau, A.A.Abrikosov and I.M.Khalatnikov, Doklady AN USSR 95 (1954) 497,773,1177, 96 (1954) 261.

[41] A.A.Abrikosov, A.D.Galanin and I.M.Khalatnikov, Doklady AN USSR, 97 (1954) 793.

[42] A.D.Galanin, B.L.Ioffe and I.Ya.Pomeranchuk, ZhETF 29 (1955) 51.

[43] L.D.Landau and I.Ya.Pomeranchuk, Doklady AN USSR 102 (1955) 489.

[44] V.V.Sudakov, ZhETP 30 (1956) 87. 
[45] V.G.Gorshkov, V.N.Gribov and G.V.Frolov, ZhETF 51 (1966) 1093.

[46] V.G.Gorshkov, V.N.Gribov, L.N.Lipatov and G.V.Frolov, Yad.Fiz. 6 (1967) 129, 361.

[47] B.L.Ioffe, V.A.Khoze and L.N.Lipatov, Hard Processes, North Holland, 1984.

[48] A.I.Akhiezer and V.B.Berestetsky, Kvantovaya elektrodinamika [Quantum Electrodynamics], 3-rd Edition, Nauka, Fizmatlit, Moscow, 1969.

[49] V.B.Berestetsky, E.M.Lifshitz and L.P.Pitaevsky, Reliativistskaya kvantovaya teoriya [Relativistic Quantum Theory], part 1, Nauka, Fizmatlit, Moscow 1968.

[50] T.D.Lee and C.N.Yang, Phys.Rev.104 (1956) 254.

[51] W.Pauli, in: Niels Bohr and the Development of Physics, Pergamon Press, London, 1955.

[52] B.L.Ioffe, ZhETF, 32 (1957) 1246 (Appendix, paper V).

[53] B.L.Ioffe, L.B.Okun and A.P.Rudik, ZhETF, 32 (1957) 396 (Appendix, paper IV).

[54] L.D.Landau, ZhETF 32 (1957) 405.

[55] L.D.Landau, ZhETF 32 (1957), 407.

[56] C.S.Wu et al, Phys.Rev. 105 (1957) 1413.

[57] T.D.Lee, R.Oeheme and C.N.Yang, Phys.Rev. 106 (1957) 340.

[58] J.H.Christenson, J.W.Cronin, V.L.Fitch and R.Turley, Phys.Rev.Lett. 13 (1964) 138.

[59] V.B.Berestetsky, B.L.Ioffe, A.P.Rudik and K.A.Ter-Martirosyan, Phys.Rev 111 (1958) 522 .

[60] L.B.Okun and B.M.Pontecorvo, ZhETF 32 (1957) 1587.

[61] R.P.Feynman and M.Gell-Mann, Phys.Rev. 109 (1958) 193.

[62] R.E.Marshak and E.C.G.Sudarshan, Phys.Rev. 109 (1958) 1860.

[63] S.S.Gershtein and Ya.B.Zeldovich, ZhETF 29 (1955) 698.

[64] M.Gell-Mann, Phys.Rev. 111 (1958) 362.

[65] V.G.Vaks and B.L.Ioffe, ZhETF 35 (1958) 221, Nuovo Cim. 10 (1958) 342.

[66] K.A.Ter-Martirosyan, ZhETF 22 (1952) 284.

[67] G.V.Skornyakov and K.A.Ter-Martirosyan, ZhETF 31 (1956) 775.

[68] I.Ya.Pomeranchuk and E.L.Feinberg, Doklady AN USSR, 93 (1953) 439.

[69] E.L.Feinberg and I.Ya.Pomeranchuk, Nuovo Cim. Suppl. No.4 (1956) 652.

[70] A.I.Akhiezer and I.Ya.Pomeranchuk, Physics Uspekhi, 55 (1958) 593. 
[71] E.Fermi, Progr.Theor.Phys. 5 (1950) 570.

[72] I.Ya.Pomeranchuk, Doklady AN USSR 78 (1951) 889 (Appendix, paper II).

[73] L.D.Landau and S.Z.Belenky, Physics Uspekhi 56 (1955) 309.

[74] E.L.Feinberg, Journ.of Phys. 5 (1941) 177.

[75] B.L.Ioffe, A.P.Rudik and I.M.Shmushkevich, Doklady AN USSR, 77 (1951) 403, ZhETF 22 (1952) 11, 21.

[76] I.Ya.Pomeranchuk, Doklady AN USSR, 80 (1951) 53, ZhETF 22 (1952) 129.

[77] I.Ya.Pomeranchuk, ZhETF 30 (1956) 423.

[78] L.B.Okun and I.Ya.Pomeranchuk, ZhETF 30 (1956) 424.

[79] L.B.Okun and I.Ya.Pomeranchuk, ZhETF 36 (1959) 300, Nucl.Phys. 10 (1959) 492.

[80] I.Ya.Pomeranhuk, ZhETF 34 (1958) 725.

[81] V.Ya.Fainberg and E.S.Fradkin, Doklady AN USSR 109 (1956) 507.

[82] B.L.Ioffe, ZhETF 31 (1956) 583.

[83] A.I.Akhiezer and I.Ya.Pomeranchuk, Nekotorye problemy teorii yadra [Some problems of the theory of nucleous], Gostechizdat, 1-st edition 1948, 2-nd edition - 1950.

[84] B.Wentzel, Zs.f.Phys. 86 (1993) 479, 635; 87 (1934) 726.

[85] P.A.M.Dirac, Ann.de l'Inst.Poincare, 9 (1939) 13.

[86] I.Pomeranchuk, Phys.rev. 76 (1949) 298. 


\title{
Appendix
}

In the Appendix are presented few papers, done at ITEP, published in Russian at the years 1944-1957 and, perhaps, unknown or almost unknown in the West.

\section{On the maximal energy, reachable in betatron 3}

\author{
D.D.Ivanenko ${ }^{1)}$ and I.Ya.Pomeranchuk $k^{2}$ \\ 1) Institute of Physics of Moscow State University \\ 2) Physico-Technical Institute of Academy of Science of USSR, Leningrad
}

Kerst [1] was constructed a new apparatus - the betatron, with which it was possible to get the flux of electrons with the energy up to $10 \mathrm{MeV}$. The principle of betatron action is: the electrons are accelerating by the electric field, arising from the varying magnetic field inside the circle, where the electrons are moving. Unlike the cyclotron, which can accelerate particles in nonrelativistic domain only, in betatron there is no limits of energies, due to dependence of particle mass from their velocities.

However, there exist another circumstance, which set the limit of the energies reachable in betatron. The origin of such limit is the bremsstrahlung of electrons, moving on circular orbit in the magnetic field. Indeed, the electron is accelerating, if it is moving in the magnetic field, and in the accord of electrodynamics, it emits the energy. It is easy to see, that because of large size of the orbit, the quantum effects plays no role here. As it was shown in [2], the electron moving in the magnetic field $H$, is emitting the energy, equal to

$$
-\frac{d E}{d x}=\frac{2}{3}\left(\frac{e^{2}}{m c^{2}}\right)^{2}\left(\frac{E}{m c^{2}}\right)^{2}\left[\frac{\mathbf{v H}}{c}\right]^{2}
$$

on the unit of length. (Here $e$ and $m$ are the charge and mass of electron, $\mathbf{v}$ and $\mathbf{E}$ are its velocity and energy. At the derivation of (11) it was assumed, that $E \gg m c^{2}$.) In the Kerst's betatron on the main part of the electron way $v \approx c$ and the direction of the velocity is perpendicular to $H$. Therefore

$$
-\frac{d E}{d x}=\frac{2}{3} r_{0}^{2}\left(\frac{E H}{m c^{2}}\right)^{2},\left(r_{0}=\frac{e^{2}}{m c^{2}}\right)
$$

The limiting energy $E_{0}$ is determined from the condition, that (2) is equal to the energy gain of electron on the unit of length [3]:

$$
\frac{2}{3} r_{0}^{2}\left(\frac{E_{0} H}{m c^{2}}\right)^{2}=\frac{e\left|\frac{d \Phi}{d t}\right|}{2 \pi R_{0} c}=e R_{0}|\dot{H}|
$$

(Here $R_{0}$ is the radius of the orbit, $\Phi$ - is the induction flux, $\dot{H}=d H / d t$ ). It follows from (3)

$$
E_{0}=m c^{2} \sqrt{\frac{2}{3} \frac{e}{c}\left|\frac{R_{0} \dot{H}}{r_{0}^{2} H^{2}}\right|} .
$$

\footnotetext{
${ }^{3}$ Doklady of AN USSR, 44 1944, 343.
} 
The magnitude of $H, \dot{H}$ and $E$, which are used now, give the numerical value of $E_{0} \approx 5 \cdot 10^{8}$ $\mathrm{eV}$. This number is only few times larger, than the value of energy, $E \approx 10^{8} \mathrm{eV}$, which is proposed to get in the betatron under construction.

According to (4), $E_{0}$ is inversally proportional to the magnetic field strength and is proportional to the square root of the energy gain on the unit of length. Therefore, in order to increase $E_{0}$ it is necessary to go to smaller $H$ and larger frequencies.

The radiation in the magnetic field also influence the bunch focusing, since the electron energy is increasing more slowly with increasing of $H$ in comparison with the case, when the radiation dumping is not accounted. This problem, however, requires special consideration.

\section{References}

[1] D.W.Kerst, Phys. Rev. 61, (1942) 93.

[2] I.Pomeranchuk, J.Phys. USSR, 2, 1940, 65.

[3] D.W.Kerst, R.Serber, Phys.Rev. 80, 1941, 53.

Received 20.01.1944 


\title{
II To the theory of multiparticle production in one event 4
}

\author{
I.Ya.Pomeranchuk
}

Academy of Science of USSR

As is known [1, 2], the nucleon-meson interaction, responsible for nuclear forces, is very strong: $\pi$-mesons are demonstrating the strong interaction with nucleons [3, 4]. As a consequence, the application of the meson theory to the description of nucleon-meson interaction meets the enormous difficulties, since the perturbation theory in not applicable here (may be with exception of low energy domain). The nonapplicability of perturbation theory is especially evident at high energies, where the collisions with many particles production play the main role (the showers production).

This conclusion directly follows from the fact $\pi$-mesons (charged and neutral) are pseudoscalar particles [5, 6], which interaction energy with nucleons has the form

$$
U=g \bar{\psi} \gamma_{5} \psi \varphi+f \frac{\hbar}{\mu c} \varepsilon_{i k l m} \bar{\psi} \gamma_{i} \gamma_{k} \gamma_{l} \psi \frac{\partial \varphi}{\partial x_{m}}
$$

The constant $g^{2} / \hbar c$ is not small and under the action of the first term the probability of the processes of the many particle production in one event is large, since the ratio of the probability of $n$ particles production to the ones of $n-1$ particles is of the order of $g^{2} / \hbar c$. Under the action of term, proportional to $f$, this statement becomes even stronger, because the interaction increases with energy [7]. Therefore, the consideration of nucleon-nucleon or nucleon- $\pi$-meson collisions requires the methods, principially different from perturbation theory.

Recently, Fermi [8] paids attention to the possibly of using the methods of statistical mechanics and thermodynamics to the description of nuclear collisions at high energies. This possibility is a direct consequence of intensive interaction of colliding and produced particles. According to Fermi the energy of colliding particles is secreted in a small volume, in which the statistical equilibrium is setted, where the probabilities of various states are proportional to their statistical weights. By choosing this volume equal to

$$
\Omega=\Omega_{0} \frac{2 M c^{2}}{W}=\frac{4 \pi}{3}\left(\frac{\hbar}{\mu c}\right)^{3} \frac{2 M c^{2}}{W}
$$

( $M$ and $\mu$ are the nucleon (and pion) masses, $W$ is the energy in c.m. system) Fermi calculated the probabilities of showers production as a function of the number of produced particles and their masses at various initial energies. (The total cross section Fermi assumed to be equal $\left.\pi(\hbar / \mu c)^{2} .5\right)$

However, the value $\Omega(\mathbb{1})$ looks not correct in the domain of relativistic energies, where many particles are produced in the collision. Each produced particle (nucleon, $\pi$-meson etc.) is strongly interacting with other particles. Therefore, if $\Omega$ is chosen, as given by (1), then in the domain of the size of order $\hbar / \mu c$ many particles occur. All these particles are strongly interacting with one another and it is nonlegitimate to use for the analysis the statistical

\footnotetext{
${ }^{4}$ Doklady AN USSR 78, 1951, 889.

${ }^{5}$ Perhaps, this value is to large. The cosmic rays data indicated that its magnitude is equal to $(\hbar / \mu c)^{2}$.
} 
weights and thermodynamical formulae of ideal gases, as it was done in [8]. Instead of this it is necessary to accept, that each particle is continuing the interaction with all other, what results to the strongly increasing of the number of produced particles. The size of the whole system will strongly increasing. The expansion in all directions proceeds with the velocity of light. This process will continue till the distances between the particles will be of order $\sqrt{\sigma} \approx \sqrt{\hbar^{2} / \mu^{2} c^{2}}$. After that the particles will move without interaction. At the final state the critical density $n_{0}$

$$
n_{0} \approx\left(\frac{\mu c}{\hbar}\right)^{3}
$$

could correspond to various particles number $N$, i.e. to the various volume

$$
\Omega \approx \frac{N}{n_{0}} \approx\left(\frac{\hbar}{\mu c}\right)^{3} N
$$

Unlike to (11) $\Omega$ is not decreasing with the increase of energy, but even increasing with the number $N$ of particles in the shower. The probabilities $P(N)$ of various $N$ can be obtained by using (3) and eq.(13) in [8]:

$$
P(N) \approx\left[\left(\frac{4 \pi}{3}\right)^{1 / 3} \frac{1}{\pi^{2 / 3}} \frac{W}{\mu c^{2}}\right]^{3 N} \frac{N^{N}}{(3 N) !\left[\left(\frac{N}{3}\right) !\right]^{3}} .
$$

An additional factor $N^{N}$ arises from (3) and the factor $[(N / 3) !]^{3}$ accounts for nonindification of $\pi^{-}, \pi^{+}$and $\pi^{0}$. In calculation of (4) it was supposed, that $N \gg 1$ and $N_{\pi^{+}}=N_{\pi^{-}}=N_{\pi^{0}}$. The nucleon pairs are not accounted in (44) and all particles are considered as ultrarelativistic.

The maximum of $P$ is reached at $\bar{N}$ equal

$$
\bar{N} \approx \frac{W}{\varepsilon}=\frac{\sqrt{2 M c^{2} W^{\prime}}}{\varepsilon},
$$

where $\varepsilon$ is of order of few hundred of $\mathrm{MeV}, W^{\prime}$ - is the energy in the laboratory coordinate system, $W^{\prime} \gg M c^{2}$. Therefore, the mean number of particles in the shower is increasing proportionally to $\sqrt{W^{\prime}}$, but not $\sqrt[4]{W^{\prime}}$, as it was obtained in [8].

The equation (5) is applicable in the case of central collisions, when the whole energy of colliding particles is going to the produced ones. If the collision is not central, the number of produced particles will be much less, than given by (5).

The temperature of the system at the moment, when the critical density (2) is achieved, is of order $\varepsilon$, i.e. it is less, than $M c^{2}$. Therefore, even at very high energies, the final temperature are smaller, then the temperatures needed for nucleon pair production. As a consequence in the star observed in [9] it should not be nucleon pairs unlike the conclusion done in [8].

Developing the method presented in [8], let us mention, that in nucleon-antinucleon collision the dominating process is the nucleon-antinucleon annihilation, into pions. This is caused bye the fact, that the statistical weight of state, where the nucleon pair is absent is much larger, then the weight, where such pair persist. Independently of initial energy, the number of nucleon pairs in all showers shall be much smaller, than the number of $\pi$-mesons. The mean energy of particles in such showers (in c.m.s.) shall be independent of initial energy and to be order $\varepsilon$.

In the showers, which are caused by noncentral collisions, the number of produced particles is much smaller, than $\bar{N}$. Such shower consist of the showers, developing around each 
of colliding particles. However, even when $N \ll \bar{N}$, the mean energy of produced particles in each of the showers shall to be of the order of $\varepsilon$ in the coordinate system, where total moment of the shower is zero. In this system the distribution of particles is isotropic.

I am indebted to L.D.Landau and Ya.B.Zeldovich for useful discussions.

Received 01.04.1954

\section{References}

[1] G.Wentzel, Rev.Mod.Phys. 19, 1974, 1.

[2] W.Pauli, The meson theory of nuclear forces, 1947, Gostechizdat, Moscow, p.75.

[3] K.A.Brueckner, Phys.Rev. 79, 1950, 641.

[4] J.Ashkin, A.Simon, R.Marshak, Progr.Theor.Phys.5, 1950, 634.

[5] L.Aamodt, J.Hadley, W.Panofsky, Phys.Rev. 80, 1950, 282.

[6] S.Tamor, R.E.Marshak, Phys.Rev. 80, 1950, 766.

[7] H.W.Lewis, J.R.Oppenheimer, S.A.Wouthuysen, Phys.Rev. 73, 1948, 127.

[8] E.Fermi, Progr.Theor.Phys. 5, 1950, 570.

[9] J.J.Lord, J.Fainberg, M.Schein, Phys.Rev. 80, 1950, 970. 


\section{The heat conductivity of the completely ionized gas at high temperatures 6}

\section{V.B.Berestetsky, B. L. Ioffe and I.Ya.Pomeranchuk}

We consider the heat conductivity of the completely ionized gas at the temperatures of order $100 \mathrm{KeV}$. At such condition the velocities of ions are small in comparison with electron velocities and the contribution of ions to heat conductivity can be neglected. The kinetic (Boltzmann) equation for the electron distribution function in the general case, when persist the temperature gradient and the external electric field $\mathbf{E}$ can be written as:

$$
(V \nabla n)-\frac{\partial n}{\partial \mathbf{p}} e \mathbf{E}=-J_{i e}-J_{e e},
$$

where $J_{i e}$ is the collisions integral of electrons with ions and $J_{e e}$ is the integral of $e e$ collisions.

$$
\begin{gathered}
J_{i e}=\int v\left[N(\mathbf{P}, \mathbf{r}) n(\mathbf{p}, \mathbf{r})-N\left(\mathbf{P}^{\prime}, \mathbf{r}\right) n\left(\mathbf{p}^{\prime}, \mathbf{r}\right)\right], \sigma_{s}\left(p, \theta^{\prime}\right) d \Omega d \mathbf{P}, \\
J_{e e}=\int\left[n(\mathbf{P}, \mathbf{r}) n(\mathbf{p}, \mathbf{r})-n\left(\mathbf{P}^{\prime}, \mathbf{r}\right) n\left(\mathbf{p}^{\prime}, \mathbf{r}\right)\right] d w\left(\mathbf{p}, \mathbf{p}^{\prime}, \mathbf{P}\right) d \mathbf{P},
\end{gathered}
$$

Here $N(\mathbf{P}, \mathbf{p})$ is the ion distribution function, $\sigma_{s}\left(p, \theta^{\prime}\right) d \Omega^{\prime}$ is the differential cross section of electron-ion collisions and $w$ is the probability of the scattering at the ee collisions.

As is known [1], at the kinetical processes in gases in case of Coulomb interaction the main role are playing the collisions with large impact parameters, corresponding to small momentum transfers. For such collisions, i.e. for the collisions, in which the momentum transfer $q=\left|\boldsymbol{\rho}^{\prime}-\mathbf{p}\right| \ll p$ is much smaller, then the value of the momentum $p$, the cross section of electron scattering on the ion is equal

$$
\sigma_{s}(p, 0)=4\left(\frac{e^{2}}{p v}\right)^{2} \frac{1}{\theta^{4}}
$$

where $\theta$ is the scattering angle and the ion charge was putten to 1 . The scattering probability at the collision of relativistic electrons can be found from the scattering matrix element, which is calculated using the relativistic wave function with account of retardation [2]:

$$
H_{A E}=4 \pi c^{2} e^{2}\left\{\frac{\left(u_{01}^{*} u_{1}\right)\left(u_{02}^{*} u_{2}\right)-\left(u_{01}^{*} \alpha_{1} u_{1}\right)\left(u_{02}^{*} \alpha_{2} u_{2}\right)}{c^{2}\left(\mathbf{p}_{01}-\mathbf{p}_{1}\right)^{2}-\left(E_{01}-E_{1}\right)^{2}}\right\},
$$

In (5) $u$ - are electron spinors, $p$ - are the momenta, $E$ - are the electron energies, the indexes 1 and 2 refer to the first and second electrons, the index 0 denotes the initial states. In the matrix element (5) the exchange effects are not accounted and there is no antisymmetrization over the final states. But in the collisions, where the impact parameters $d$ are much larger, then the electron wave length $\lambda, d \gg \lambda$, the quantum mechanical exchange effect (i.e. the interference terms) can be disregarded. The classical factor 2 will be accounted in the final formulas, where the integration will be performed over the whole space instead of the half of space, as it should be done for identical particles. At small momentum transfer we can put $u_{01}=u_{1}$ and $u_{02}=u_{2}$ and therefore

$$
u_{01}^{*} u_{1}=1, \quad u_{01}^{*} \alpha u_{1}=\frac{\mathbf{v}_{1}}{c},
$$

\footnotetext{
${ }^{6}$ This work was performed in ITEP in 1951 and was classified. Declassified in 1972 and published in: I.Ya.Pomeranchuk, [Sobranie trudov], Nauka, Moscow, 1972.
} 


$$
u_{02}^{*} u_{2}=1, \quad u_{02}^{*} \alpha u_{2}=\frac{\mathbf{v}_{2}}{c}
$$

Let us expand in powers of

$$
\mathbf{q}=\mathbf{p}_{1}-\mathbf{p}_{01}
$$

the energies difference in the denominator of (15)

$$
E_{1}-E_{01}=\mathbf{v}_{1} \mathbf{q}
$$

Then the probability of the scattering of electron with momentum $\mathbf{p}_{\mathbf{0 1}}$ into the element $d \mathbf{p}_{1}$ of the momentum space is equal

$$
d w=4 e^{4} \frac{\left(1-\frac{\mathbf{v}_{01} \mathbf{v}_{02}}{c^{2}}\right)^{2}}{\left[q^{2}-\left(\mathbf{v}_{01} \mathbf{q}\right)^{2} / c^{2}\right]^{2}} \delta\left(\mathbf{v}_{01}-\mathbf{v}_{02}, \mathbf{q}\right) d \mathbf{p}_{1} .
$$

(The argument of $\delta$-function, corresponding to energy conservation, is expanded in powers of $q$ up to first order terms.)

As usually done by the heat conductivity calculation, let us suppose that the temperature gradient as well the electric field are small. Therefore, the deviation of the electron distribution from the equilibrium Maxwell ones is small

$$
n=n_{0}(1+\chi)
$$

Here $n_{0}$ is the Maxwell distribution of relativistic electron gas [3]

$$
n_{0} d p=\rho_{e} \frac{\exp \left[-c \sqrt{m^{2} c^{2}+p^{2}} / T\right]}{2\left(\frac{T}{m c^{2}}\right)^{2} K_{1}\left(\frac{m c^{2}}{T}\right)+\frac{T}{m c^{2}} K_{0}\left(\frac{m c^{2}}{T}\right)} \frac{d \mathbf{p}}{4 \pi(m c)^{3}},
$$

where $\rho_{e}$ is the number of electrons in $\mathrm{cm}^{3}, T$-is the temperature in the units of energy, $K_{0}$ and $K_{1}$ - are the McDonald functions. The function $\chi_{1}$ describing the deviation from Maxwell distribution shall have the from

$$
\chi=\mathbf{p} \nabla T \chi_{1}(p)+\mathbf{p E} e \chi_{2}(p),
$$

up to higher order terms in $\nabla T$ and $\mathbf{E} . \chi_{1}(p)$ and $\chi_{2}(p)$ are the functions of the absolute values of the momentum. If the electron density is assumed to be constant, the left hand side of eq.(1) can be represented in the form

$$
\frac{\partial n_{0}}{\partial T}(\mathbf{v} \nabla T)-\frac{\partial n_{0}}{\partial p} e \frac{\mathbf{p E}}{p} .
$$

Let us now determine the collision integrals. Since the equations are linear relative to $\chi_{1}$ find first the equation for $\chi_{1}$. The equation for $\chi_{2}$ will be found in a similar way. Using (10) the integral of electron-ion collisions can be written as

$$
\begin{gathered}
J_{i e}=\int v\left\{N(P) n_{0}(p)[1+\chi(\mathbf{p}, \mathbf{r})]-N\left(P^{\prime}\right) n_{0}\left(p^{\prime}\right)\left[1+\chi\left(\mathbf{p}^{\prime}, \mathbf{r}\right)\right]\right\} \times \\
\times \sigma_{s}\left(p, \theta^{\prime}\right) d \Omega^{\prime} d \mathbf{P}=v n_{0}(p) \int\left[\chi(\mathbf{p}, \mathbf{r})-\chi\left(\mathbf{p}^{\prime}, \mathbf{r}\right)\right] N(P) \sigma_{s}\left(p, \theta^{\prime}\right) d \Omega^{\prime} d \mathbf{P},
\end{gathered}
$$


since in accord with energy conservation at the collision

$$
N(P) n_{0}(p)=N\left(P^{\prime}\right) n_{0}\left(p^{\prime}\right) .
$$

The integration over $\mathbf{P}$ gives

$$
\int N(P) d \mathbf{P}=\rho_{i}
$$

where $\rho_{i}$ is the number of ions in $\mathrm{cm}^{3}$. Using (12), we get

$$
J_{i e}=\rho_{i} v n_{0}(p) \nabla T \int\left(\mathbf{p}-\mathbf{p}^{\prime}\right) \chi_{1}(p) \sigma_{s}\left(p, \theta^{\prime}\right) d \Omega^{\prime} .
$$

The integral in (17) shall be proportional to $\mathbf{p}$. Multiplying the integral by $\mathbf{p}$ and substituting $\sigma_{s}$ from (4) we find the expression for the collision integral of electrons with ions

$$
J_{i e}=4 \pi L v \rho_{i} n_{0}(p)\left(\frac{e^{2}}{p v}\right)^{2}(\mathbf{p} \nabla T) \chi_{1}(p) .
$$

In (18)

$$
L=\int_{\theta_{\min }}^{\theta_{\max }} \frac{d \theta}{\theta}=\ln \frac{r_{\max }}{r_{\min }}
$$

where $r_{\max }$ and $r_{\min }$ - are the maximal and minimal impact parameters. The maximal impact parameter is chosen [1] as the distance, where the screening of the Coulomb field of the given ion by neighbouring ions becomes important. In the absence of magnetic forces this distance can be taken to be equal to the Debye-Hückel radius

$$
r_{\min }=\sqrt{\frac{T}{4 \pi e^{2}} \frac{1}{\rho_{i}+\rho_{e}}} .
$$

The account of magnetic forces do not change the order of magnitude of $r_{\max }$ and, consequently, up to the small number of order $1 / L$, will not charge the value of the logarithm in (19). In the case of high temperature of electrons the minimal impact parameter shall to be taken equal to $\lambda=\hbar / p$, since at such distances the momentum transfers are not small. Therefore

$$
L=\frac{1}{2} \ln \left[\frac{137}{8 \pi}\left(\frac{m c}{\hbar}\right)^{3} \frac{1}{\rho_{e}}\left(\frac{\rho}{m c}\right)^{2} \frac{T}{m c^{2}}\right] .
$$

The account of only far separated collisions, which results to cut-off of logarithmically divergent integrals at $\theta_{\min }$ (or $r_{\max }$ ) restricts the accuracy of formulae (18), (26) by the value of order $\sim 1 / L \sim 0.1$ At the same order is the accuracy of the kinetical equations (43), (45) and the value of heat conductivity, presented in Table 1.

For the calculation of collisions integral in case of ee collision we use the general expression, which was obtained in [1] as a result of expansion at small momentum transfer:

$$
\begin{gathered}
J_{e e}=\frac{\partial j_{i}}{\partial x_{i}}, \quad i=1,2,3 \\
j_{i}=\frac{1}{2} \int d \mathbf{P}\left[n(\mathbf{p}) \frac{d n(\mathbf{P})}{\partial P_{k}}-n(\mathbf{P}) \frac{\partial n(\mathbf{p})}{\partial p_{k}}\right] q_{i} q_{k} d w
\end{gathered}
$$


In (23) $d w$ is given by (99) (in the notation $v_{01}=\mathbf{v}, \mathbf{v}_{02}=\mathbf{V}, \mathbf{p}_{01}=\mathbf{p}, \mathbf{p}_{1}=\mathbf{p}^{\prime}, \mathbf{q}=\mathbf{p}^{\prime}-\mathbf{p}$ ). By substitution of the expansion (10) for $n(\mathbf{p})$ in (23) and by accounting only the terms linear in $\chi$, we get for $j_{i}$

$$
j_{i}=-\frac{1}{2} n_{0} \int n_{0}(P)\left[\frac{\partial \chi(\mathbf{p})}{\partial p_{k}}-\frac{\partial \chi(\mathbf{P})}{\partial P_{k}}\right] q_{i} q_{k} d w d \mathbf{P}
$$

The equilibrium function $n_{0}$ do not contribute to $j_{i}$.

The substitution of $\partial \chi_{1} / \partial p_{k}$, given by (12)

$$
\frac{\partial \chi_{1}}{\partial p_{k}}=\chi_{1}(p) \frac{\partial T}{\partial x_{k}}+(\mathbf{P} \nabla T) \frac{p_{k}}{p} \frac{\partial \chi_{1}}{\partial p}
$$

and after transformation the resulting expression to the form convenient for angular integration, it is obtained the formula for collision integral in case of ee collisions

$$
\begin{gathered}
J_{e e}=\frac{1}{2}(\mathbf{p} \nabla T) \frac{1}{p^{2}}\left\{\left[n_{0}\left(\Phi_{2}-3 \Phi_{1}\right)-p \frac{\partial}{\partial p}\left(n_{0}(p) \Phi_{1}(p)\right)\right] \chi_{1}(p)-\right. \\
-\left[4 n_{0}(p) p \Phi_{1}(p)+p^{2} \frac{\partial}{\partial p}\left(n_{0}(p) \Phi_{1}(p)\right)\right] \frac{\partial \chi_{1}}{\partial p}- \\
-n_{0}(p) \Phi_{1}(p) p^{2} \frac{\partial^{2} \chi_{1}}{\partial p^{2}}-n_{0}(p)\left[\bar{\Phi}_{2}(p)-3 \bar{\Phi}_{1}(p)\right]+ \\
\left.+p \frac{\partial}{\partial p}\left[n_{0}(p) \bar{\Phi}_{1}(p)\right]+3 n_{0} \bar{\Phi}_{3}(p)+p \frac{\partial}{\partial p}\left[n_{0}(p) \bar{\Phi}_{3}(p)\right]-n_{0}(p) \bar{\Phi}_{1}^{*}(p)\right\},
\end{gathered}
$$

where

$$
\begin{gathered}
\Phi_{1}(p)=\int n_{0}(P)\left(\frac{\mathbf{q p}}{p}\right)^{2} d \mathbf{P} d w \\
\Phi_{2}(p)=\int n_{0}(P) q^{2} d \mathbf{P} d w \\
\bar{\Phi}_{1}(p)=\int n_{0}(p)\left(\frac{\mathbf{q} \mathbf{p}}{p}\right)^{2} \chi_{1}(P) d \mathbf{P} d w \\
\bar{\Phi}_{2}(p)=\int n_{0}(P) q^{2} \chi_{1}(P) d \mathbf{P} d w \\
\bar{\Phi}_{3}(p)=\int \frac{\mathbf{P} \mathbf{p}}{p} n_{0}(P) \frac{\partial \chi_{1}(P)}{\partial P} \frac{v}{V}\left(\frac{\mathbf{q} \mathbf{p}}{p}\right)^{2} d \mathbf{P} d w \\
\bar{\Phi}_{1}^{*}(p)=\int n_{0}(P) P \frac{\partial \chi_{1}(P)}{\partial P}\left(\frac{v}{V}\right)^{2}\left(\frac{\mathbf{q p}}{p}\right)^{2} d \mathbf{P} d w .
\end{gathered}
$$

At the derivation of (26) were accounted the equalities

$$
\mathbf{V q}=\mathbf{v q}, \frac{\mathbf{P q}}{P}=\frac{v}{V} \frac{\mathbf{p q}}{p}
$$

caused by the presence of $\delta$-function in (9). Let us perform the integration in

$$
\Phi_{1}(p)=4 e^{4} \int n_{0}(P)\left(\frac{\mathbf{q p}}{p}\right)^{2} \frac{\left(1-\frac{1}{c^{2}} \mathbf{v} \mathbf{V}\right)^{2}}{\left[q^{2}-\frac{1}{c^{2}}(\mathbf{v q})^{2}\right]^{2}} \delta(\mathbf{v}-\mathbf{V}, \mathbf{q}) d \mathbf{q} d \mathbf{P} .
$$


First integrate over the directions of the vector $\mathbf{P}$, after that over all values of $\mathbf{q}$. It is convenient for the integration over the directions of $\mathbf{P}$ to introduce the coordinate system with the $z$-axe along $\mathbf{q}$. In this system the unit vectors $\mathbf{P} / P$ and $\mathbf{p} / p$ can be represented as

$$
\begin{aligned}
& \frac{\mathbf{P}}{P}=\{\sin \beta \cos \delta ; \quad \sin \beta \sin \delta ; \quad \cos \beta\}, \\
& \frac{\mathbf{p}}{p}=\{\sin \theta \cos \varphi ; \quad \sin \theta \sin \varphi ; \quad \cos \theta\},
\end{aligned}
$$

and the integral takes the form

$$
\begin{gathered}
\Phi_{1}(p)=4 e^{4} \int_{0}^{\infty} n_{0}(P) P^{2} d P \int \frac{d q}{q} \int \frac{\cos ^{2} \theta d \cos \theta d \varphi}{\left(1-\frac{\nu^{2}}{c^{2}} \cos ^{2} \theta\right)^{2}} \times \\
\times\left[1-\frac{1}{c^{2}} V v(\cos \beta \cos \theta+\sin \beta \sin \theta \cos (\varphi-\delta)]^{2} \delta(v \cos \theta-V \cos \beta) d \cos \beta d \delta .\right.
\end{gathered}
$$

The integration over $\beta, \delta$ and $\varphi$ gives

$$
\begin{aligned}
& \Phi_{1}(p)=16 \pi^{2} e^{4} \int_{0}^{\infty} n_{0}(P) P^{2} \frac{1}{V} d P \int \frac{d q}{q} \int_{0}^{\lambda} \frac{\cos ^{2} \theta d \cos \theta}{\left(1-\frac{\nu^{2}}{c^{2}} \cos ^{2} \theta\right)^{2}} \times \\
& \quad \times\left\{\left(1-\frac{v^{2}}{c^{2}} \cos ^{2} \theta\right)^{2}+\frac{V^{2} v^{2}}{2 c^{2}} \sin ^{2} \theta-\frac{v^{4}}{2 c^{4}} \sin ^{2} \theta \cos ^{2} \theta\right\},
\end{aligned}
$$

where

$$
\lambda=\left\{\begin{array}{ccc}
\frac{v}{V} & \text { at } & v>V, \\
1 & \text { at } & v<V .
\end{array}\right.
$$

Like the case of the collisions of electrons with ions the integral over momentum transfer is equal

$$
\int \frac{d q}{q}=\ln \frac{q_{\max }}{q_{\min }}=\ln \frac{r_{\max }}{r_{\min }}=L
$$

Using the notation $\cos \theta=u$ and $\beta=v / c$ we have finally

$$
\begin{aligned}
& \Phi_{1}(p)=16 \pi^{2} L e^{4} \int_{0}^{\infty} n_{0}(P) \frac{P^{2}}{V} d P \int_{0}^{\lambda} \frac{u^{2} d u}{\left(1-\beta^{2} u^{2}\right)^{2}} \times \\
& \times\left\{\left(1-\beta^{2} u^{2}\right)^{2}+\beta^{2} \frac{V^{2}}{2 c^{2}}\left(1-u^{2}\right)-\frac{1}{2} \beta^{4} u^{2}\left(1-u^{2}\right)\right\} .
\end{aligned}
$$

For $\Phi_{2}$ and $\Phi_{3}$ in a similar way we find

$$
\begin{aligned}
& \Phi_{2}(p)=16 \pi^{2} L e^{4} \int_{0}^{\infty} n_{0}(P) \frac{P^{2}}{V} d P \int_{0}^{\lambda} \frac{d u}{\left(1-\beta^{2} u^{2}\right)^{2}} \times \\
& \times\left\{\left(1-\beta^{2} u^{2}\right)^{2}+\beta^{2} \frac{V^{2}}{2 c^{2}}\left(1-u^{2}\right)-\frac{1}{2} \beta^{4} u^{2}\left(1-u^{2}\right)\right\},
\end{aligned}
$$




$$
\begin{gathered}
\bar{\Phi}_{3}(p)=16 \pi^{2} L e^{4} \int_{0}^{\infty} n_{0}(P) \frac{v^{2}}{V^{3}} P^{3} d P \frac{\partial \chi_{1}(P)}{\partial P} \int_{0}^{\lambda} \frac{u^{2} d u}{\left(1-\beta^{2} u^{2}\right)^{2}} \times \\
\times\left\{u^{2}\left(1-\beta^{2} u^{2}\right)^{2}-\frac{3}{2}\left(1-u^{2}\right)\left(1-\beta^{2} u^{2}\right)\left(\frac{V^{2}}{c^{2}}-\beta^{2} u^{2}\right)+\frac{1}{2}\left(1-u^{2}\right)\left(\frac{V^{2}}{2 c^{2}}-\beta^{2} u^{2}\right)\right\} .
\end{gathered}
$$

After substitution of (39),(40),(41) and the expressions for $\Phi_{1}, \bar{\Phi}_{2}$ and $\bar{\Phi}_{3}$ in the collision integral, performing the integration by parts, and using the variable

$$
x=\frac{c p}{T},
$$

we get the following equation for the distribution function $\chi_{1}(x)=4 \pi\left(e^{2} / c\right) \rho_{e} L \chi_{1}(p)$ :

$$
1-\frac{3 \alpha K_{0}(\alpha)+6 K_{1}(\alpha)+\alpha^{2} K_{1}(\alpha)}{\left[\alpha K_{0}(\alpha)+2 K_{1}(\alpha)\right] \sqrt{\alpha^{2}+x^{2}}}=\hat{M} \chi_{1}(x),
$$

where $\alpha=m c^{2} / T$ and the linear integro-differential operator $\hat{M}$ is defined by

$$
\begin{gathered}
\hat{M} \chi_{1}(x)=-\frac{\sqrt{\alpha^{2}+x^{3}}}{x^{3}} \chi_{1}(x)-\frac{1}{\alpha^{2} K_{0}(\alpha)+2 \alpha K_{1}(\alpha)} \frac{1}{x^{2}} \times \\
\times\left\{A(x) \chi_{1}(x)+B(x) \frac{d \chi_{1}(x)}{d x}+C(x) \frac{d^{2} \chi_{1}(x)}{d x^{2}}+\int_{0}^{\infty} K(x, y) \chi_{1}(y) d y\right\} .
\end{gathered}
$$

The coefficients $A(x), C(x)$ and the kernel $K(x, y)$ are given below

$$
\begin{gathered}
A(x)=-\frac{x^{2} \alpha^{2}}{\sqrt{x^{2}+\alpha^{2}}} e^{-\sqrt{\alpha^{2}+x^{2}}}+\frac{1}{2} e^{-\sqrt{\alpha^{2}+x^{2}}}\left\{\sqrt { \alpha ^ { 2 } + x ^ { 2 } } \left(\alpha^{2}+x^{2}+\right.\right. \\
\left.\left.+2 \sqrt{\alpha^{2}+x^{2}}+2\right)\left[1-\frac{1}{2 \beta}\left(1-\beta^{2}\right) \ln \frac{1+\beta}{1-\beta}\right]-\alpha^{2}\left(1-\frac{x}{2 \beta}\right)\left[3-\frac{1}{2 \beta}\left(3-\beta^{2}\right) \ln \frac{1+\beta}{1-\beta}\right]\right\}+ \\
+\frac{1}{2} \int_{0}^{x} y \sqrt{\alpha^{2}+y^{2}} e^{-\sqrt{\alpha^{2}+y^{2}}} d y\left\{-\frac{1}{\beta}\left[\gamma\left(1-\beta^{2}\right)-\right.\right. \\
\left.-\frac{1}{2}\left(3-\beta^{2}\right)\left(1-\gamma^{2}\right) \ln \frac{1+\gamma}{1-\gamma}\right]+\frac{x}{2 \beta^{2}}\left[\gamma\left(5-3 \beta^{2}\right)-\right. \\
B(x)=-\frac{x}{4} e^{-\sqrt{\alpha^{2}+x^{2}}}\left\{\frac{\alpha^{2}}{\beta^{2}}\left[3+7 \beta^{2}-\frac{1}{2 \beta}\left(3+6 \beta^{2}-\beta^{4} \ln \frac{1+\beta}{1-\beta}\right)\right]-\right. \\
-\frac{\alpha^{2} x}{\beta}\left(3-\frac{3-\beta^{2}}{2 \beta} \ln \frac{1+\beta}{1-\beta}\right)+2\left(\alpha^{2}+x^{2}+2 \sqrt{\alpha^{2}+x^{2}}+2\right) \times \\
\times\left[\frac{1}{\beta^{2}}\left(1+\frac{1+\gamma}{1-\gamma}\right]\right\} ; \\
-\frac{x}{4} \int_{0}^{x} y \sqrt{\alpha^{2}+y^{2}} e^{-\sqrt{\alpha^{2}+y^{2}}} d y\left\{\frac { 1 } { \beta ^ { 3 } } \left[\gamma\left(5-3 \beta^{4}+6 \beta^{2}\right)-\right.\right. \\
\left.\left.\left.\ln \frac{1+\beta}{1-\beta}\right)-\frac{x}{\beta}\left(1-\frac{1-\beta^{2}}{2 \beta} \ln \frac{1+\beta}{1-\beta}\right)\right]\right\}-
\end{gathered}
$$




$$
\begin{gathered}
\left.-\frac{1}{2}\left(5+6 \beta^{2}-3 \gamma^{2}-3 \beta^{4}-6 \beta^{2} \gamma^{2}+\beta^{4} \gamma^{2}\right) \ln \frac{1+\gamma}{1-\gamma}\right]- \\
\left.-\frac{x}{\beta^{2}}\left[\gamma\left(5-3 \beta^{2}\right)-\frac{1}{2}\left(5-3 \beta^{2}-3 \gamma^{2}+\beta^{2} \gamma^{2}\right) \ln \frac{1+\gamma}{1-\gamma}\right]\right\} \\
C(x)=-\frac{x^{2}}{4 \beta^{2}} e^{-\sqrt{\alpha^{2}+x^{2}}}\left\{2\left[1-\frac{1}{2 \beta}\left(1-\beta^{2}\right) \ln \frac{1+\beta}{1-\beta}\right]\left(\alpha^{2}+x^{2}+2 \sqrt{\alpha^{2}+x^{2}}+2\right)+\right. \\
+\alpha^{2}\left[3-\frac{1}{2 \beta}\left(3-\beta^{2}\right) \ln \frac{1+\beta}{1-\beta}\right]-\frac{x^{2}}{4 \beta^{3}} \int_{0}^{x} y \sqrt{\alpha^{2}+y^{2}} e^{-\sqrt{\alpha^{2}+y^{2}}} d y \times \\
\times\left\{\gamma\left(5-3 \beta^{2}\right)-\frac{1}{2}\left(5-3 \beta^{2}-3 \gamma^{2}+\beta^{2} \gamma^{2}\right) \ln \frac{1+\gamma}{1-\gamma}\right\} ; \\
K(x, y)=y \sqrt{\alpha^{2}+y^{2}} e^{-\sqrt{\alpha^{2}+y^{2}}}\left\{\left[\left(1-\gamma^{2}\right)\left(R_{1}+R_{2}\right)+R_{3}+R_{4}\right] \text { at } y>x,\right. \\
{\left[\left(1-\beta^{2}\right)\left(\bar{R}_{1}+\bar{R}_{2}\right)+\bar{R}_{3}+\bar{R}_{4}\right] \text { at } y<x,}
\end{gathered}
$$

where

$$
\begin{gathered}
R_{1}=-1+\frac{1-\beta^{2}}{2 \beta} \ln \frac{1+\beta}{1-\beta} \\
R_{2}=\frac{1}{2} \sqrt{\alpha^{2}+y^{2}}\left[5+\left(3 \beta^{2}-5\right) \frac{1}{2 \beta} \ln \frac{1+\beta}{1-\beta}\right], \\
R_{3}=\frac{x}{2 \beta}\left[5-4 \beta^{2}-3 \gamma^{2}+2 \beta^{2} \gamma^{2}-\frac{1}{2 \beta}\left(1-\beta^{2}\right)\left(5-3 \gamma^{2}\right) \ln \frac{1+\beta}{1-\beta}\right], \\
R_{4}=-\frac{x}{2 \beta} \sqrt{\alpha^{2}+y^{2}}\left[11-\frac{16}{3} \beta^{2}-9 \gamma^{2}+4 \beta^{2} \gamma^{2}-\right. \\
\left.-\frac{1}{2 \beta}\left(11-9 \beta^{2}-9 \gamma^{2}+7 \beta^{2} \gamma^{2}\right) \ln \frac{1+\gamma}{1-\gamma}\right] \\
\bar{R}_{1}=\frac{1}{\beta}\left[-\gamma+\frac{1}{2}\left(1-\gamma^{2}\right) \ln \frac{1+\gamma}{1-\gamma}\right] \\
\bar{R}_{2}=\frac{x}{2 \beta^{2}}\left[5 \gamma-\frac{1}{2}\left(5-3 \gamma^{2}\right) \ln \frac{1+\gamma}{1-\gamma}\right] \\
\bar{R}_{3}=\frac{\sqrt{\alpha^{2}+y^{2}}}{2 \beta}\left[\gamma\left(5-3 \beta^{2}-4 \gamma^{2}+2 \beta^{2} \gamma^{2}\right)-\frac{1}{2}\left(1-\gamma^{2}\right)\left(5-3 \beta^{2}\right) \ln \frac{1+\gamma}{1-\gamma}\right], \\
\bar{R}_{4}=-x \frac{\sqrt{\alpha+y^{2}}}{4 \beta^{2}}\left[\gamma\left(11-9 \beta^{2}-\frac{16}{3} \gamma^{2}+4 \beta^{2} \gamma^{2}\right)-\right. \\
\left.-\frac{1}{2}\left(11-9 \beta^{2}-9 \gamma^{2}+7 \beta^{2} \gamma^{2}\right) \ln \frac{1+\gamma}{1-\gamma}\right]
\end{gathered}
$$

The notation

$$
\beta=\frac{v}{c}=\frac{x}{\sqrt{x^{2}+\alpha^{2}}} ; \quad \gamma=\frac{V}{c}=\frac{y}{\sqrt{y^{2}+\alpha^{2}}} .
$$

are used. The equation for the function $\chi_{2}(x)=4 \pi\left(e^{2} / c\right) \rho_{e} L \chi_{2}(p)$ differs from (43) by the use of unhomogeneous term in the left hand side of the second term in (14) and have the form

$$
\frac{1}{\sqrt{\alpha+x^{2}}}=\hat{M} \chi_{2}(x)
$$


The border conditions for the equations (43) and (45) are: $\chi=0$ and $\chi^{\prime}(0)$ at $x=0$. These conditions are obtained automatically from the conditions of finiteness of electron-ion collision integral. This integral is dominating at small electron velocities, since the scattering cross section is inversely proportional to the 4-th power of the relative velocity of colliding particles, tends to infinity in case of electron-ion collisions and to finite limit in case of $e-e$ collisions. $\chi(x)$ is proportional to $x^{3}$, since at small, $J_{i e} \sim\left(1 / x^{3}\right) \chi(x)$. From this statement follow the mention above border conditions.

The knowledge of the distribution function allows to find easily the flux of heat and heat conductivity. The flux of heat $\mathbf{j}$ is equal

$$
\mathbf{j}=\int E_{\mathrm{kin}} \mathbf{v} n d \mathbf{p}=\int n_{0} E_{\mathrm{kin}} \mathbf{v} \chi d \mathbf{p}=\int n_{0} E_{\mathrm{kin}} \mathbf{v}\left[\mathbf{p} \nabla T \chi_{1}(p)+e \mathbf{E} \mathbf{p} \chi_{2}(p)\right] d \mathbf{p},
$$

since in case of equilibrium $\mathbf{j}$ vanishes. The electric field $\mathbf{E}$, which enters eq. (46) is caused by redistribution of charges in the gas. Such redistribution proceeds much before the equilibrium of temperatures. The electric field $\mathbf{E}$ should be determined from the condition of the vanishing of the total current

$$
\mathbf{J}=\int n \mathbf{v} d \mathbf{p}=\int n_{0} \chi \mathbf{v} d \mathbf{p}=\int n_{0} \mathbf{v}\left[(\mathbf{p} \nabla T) \chi_{1}(p)+e \mathbf{E} \mathbf{p} \chi_{2}(p)\right] d \mathbf{p}=0 .
$$

Since the unique direction in the problem in view is the direction of the gradient of the temperature, the electric field and the current have the same direction. Choosing the axe $x$ for this direction, we have from (47)

$$
e E_{x} \int n_{0}(p) v_{x} p_{x} \chi_{2}(p) d \mathbf{p}=-\frac{d T}{d x} \int n_{0}(p) v_{x} p_{x} \chi_{1}(p) d \mathbf{p} .
$$

It follows from (48)

$$
e \mathbf{E}=-\nabla T \frac{\int_{0}^{\infty} n_{0}(p) \frac{p^{4}}{E} \chi_{1}(p) d p}{\int_{0}^{\infty} n_{0}(p) \frac{p^{4}}{E} \chi_{2}(p) d p} \equiv-\zeta \nabla T .
$$

By substituting of (49) into (46), we get

$$
j_{i}=\int E_{\operatorname{kin}} n_{0}(p)\left[\chi_{1}(p)-\zeta \chi_{2}(p)\right] p_{k} v_{i} d \mathbf{p} \frac{d T}{d x_{k}} .
$$

From the other side

$$
j_{i}=-\kappa \delta_{i k} \frac{\partial T}{\partial x_{k}}
$$

where $\kappa$ is the heat conductivity. Therefore

$$
\begin{aligned}
\kappa & =-\frac{1}{3} \int E_{\operatorname{kin}} n_{0}(p)\left[\chi_{1}(p)-\zeta \chi_{2}(p)\right] \mathbf{p v} d \mathbf{p}= \\
& =-\frac{4 \pi}{3} \int_{0}^{\infty} E_{\operatorname{kin}}\left[\chi_{1}(p)-\zeta \chi_{2}(p)\right] \frac{p^{4} c^{2}}{E} d p .
\end{aligned}
$$

In terms of the variable $x$ and the functions $\chi_{1}(x), \chi_{2}(x)$ the final result looks like

$$
\kappa=-\frac{1}{12 \pi} \frac{1}{L}\left(\frac{T}{e^{2}}\right)^{2}\left(\frac{T}{m}\right)^{1 / 2} \frac{1}{\sqrt{\alpha}\left[2 K_{1}(\alpha)+\alpha K_{0}(\alpha)\right]} \times
$$




$$
\times \int_{0}^{\infty}\left(1-\frac{\alpha}{\sqrt{\alpha^{2}+x^{2}}}\right)\left[\chi_{1}(x)-\zeta \chi_{2}(x)\right] e^{-\sqrt{\alpha^{2}+x^{2}}} x^{4} d x
$$

where

$$
\zeta=\frac{\int_{0}^{\infty} e^{-\sqrt{\alpha^{2}+x^{2}}} \chi_{1}(x) \frac{x^{4}}{\sqrt{\alpha^{2}+x^{2}}} d x}{\int_{0}^{\infty} e^{-\sqrt{\alpha^{2}+x^{2}}} \chi_{2}(x) \frac{x^{4}}{\sqrt{\alpha^{2}+x^{2}}} d x}
$$

The equation for distribution function can be significantly simplified in two limits: nonrelativistic $(\alpha \gg 1)$ and ultrarelativistic $(\alpha \ll 1)$. In the nonrelativistic case it is convenient to put

$$
\begin{gathered}
x^{\prime}=\frac{1}{\sqrt{\alpha}} x=\frac{p}{\sqrt{m T}}, \\
\chi^{\prime}=\sqrt{\alpha \chi} .
\end{gathered}
$$

Being expressed in the terms of these variables the eq.'s(43) and (45) take the form

$$
\begin{aligned}
& \frac{1}{2}\left(x^{\prime 2}-3\right)=-\frac{1}{x^{\prime 3}} \chi_{1}^{\prime}\left(x^{\prime}\right)-\sqrt{\frac{2}{\pi}} \frac{1}{x^{\prime 2}}\left\{A^{\prime}\left(x^{\prime}\right) \chi_{1}^{\prime}\left(x^{\prime}\right)+B^{\prime}\left(x^{\prime}\right) \frac{d \chi_{1}^{\prime}\left(x^{\prime}\right)}{d x^{\prime}}+\right. \\
& \left.+C^{\prime}\left(x^{\prime}\right) \frac{d^{2} \chi_{1}^{\prime}\left(x^{\prime}\right)}{d x^{\prime 2}}+\int_{0}^{\infty} K^{\prime}\left(x^{\prime}, y^{\prime}\right) \chi_{1}^{\prime}\left(y^{\prime}\right) d y^{\prime}\right\} \\
& 1=-\frac{1}{x^{\prime 3}} \chi_{2}^{\prime}\left(x^{\prime}\right)-\sqrt{\frac{2}{\pi}} \frac{1}{x^{\prime 2}}\left\{A^{\prime}\left(x^{\prime}\right) \chi_{2}^{\prime}\left(x^{\prime}\right)+B^{\prime}\left(x^{\prime}\right) \frac{d \chi_{2}^{\prime}\left(x^{\prime}\right)}{d x^{\prime}}+\right. \\
& \left.+C^{\prime}\left(x^{\prime}\right) \frac{d^{2} \chi_{2}^{\prime}}{d x^{\prime 2}}+\int_{0}^{\infty} K^{\prime}\left(x^{\prime}, y^{\prime}\right) \chi_{2}\left(y^{\prime}\right) d y^{\prime}\right\}, \\
& A^{\prime}(x)=-\frac{2}{3} x^{2} e^{-\frac{x^{2}}{2}}+\frac{\sqrt{2}}{x}\left\{\Gamma\left(\frac{3}{2} ; \frac{x^{2}}{2}\right)+\frac{2}{3} \Gamma\left(\frac{5}{2} ; \frac{x^{2}}{2}\right)\right\}, \\
& B^{\prime}(x)=-\frac{x}{3}\left(4-x^{2}\right) e^{-\frac{x^{2}}{2}}-\frac{2^{3 / 2}}{3} \frac{1-x^{2}}{x^{2}} \Gamma\left(\frac{5}{2} ; \frac{x^{2}}{2}\right), \\
& C^{\prime}(x)=-\frac{x^{2}}{3} e^{-\frac{x^{2}}{2}}-\frac{2^{3 / 2}}{3} \Gamma\left(\frac{5}{2} ; \frac{x^{2}}{2}\right) \\
& K^{\prime}(x, y)= \begin{cases}x^{2} y e^{-y^{2 / 2}}\left(\frac{1}{3}-\frac{1}{5} x^{2}\right) & \text { at } y>x, \\
\frac{y^{4}}{x} e^{-y^{2 / 2}}\left(\frac{1}{3}-\frac{1}{5} y^{2}\right) & \text { at } y<x .\end{cases}
\end{aligned}
$$

The expression for heat conductivity is also simplified at $\alpha \gg 1$ (nonrelativistic case):

$$
\kappa=-\frac{1}{12 \pi} \frac{1}{\sqrt{2 \pi}} \frac{1}{L}\left(\frac{T}{e^{2}}\right)^{2}\left(\frac{T}{m}\right)^{1 / 2} \int_{0}^{\infty} x^{6}\left[\chi_{1}^{\prime}\left(x^{\prime}\right)-\zeta \chi_{2}^{\prime}\left(x^{\prime}\right)\right] e^{-\frac{x^{\prime 2}}{2}} d x^{\prime},
$$

where

$$
\zeta=\frac{\int_{0}^{\infty} e^{-\frac{x^{\prime 2}}{2}} x^{\prime 4} \chi_{1}^{\prime}\left(x^{\prime}\right) d x^{\prime}}{\int_{0}^{\infty} e^{-\frac{x^{\prime 2}}{2}} x^{\prime 4} \chi_{2}^{\prime}\left(x^{\prime}\right) d x^{\prime}} .
$$


The equations (43), (45) were solved numerically for the temperatures: $T=50 \mathrm{KeV}(\alpha=$ $10.2), T=100 \mathrm{KeV}(\alpha=5.1)$ and $T=200 \mathrm{KeV}(\alpha=2.55)$. As the control of the method it was performed the numerical solution of nonrelativistic equation (56), (57) and the solution of equations for ultrarelativistic case by Chapman-Enskog method (see below). The obtained values of $\kappa /(1 / L)\left(T / e^{2}\right)^{2}(T / m)^{1 / 2}$ are shown in Table 1 .

Table 1

\begin{tabular}{c|c|c|c|c|c}
\hline$T$ & $\begin{array}{c}T<<m c^{2} \text { (non- } \\
\text { relativ.case) }\end{array}$ & $50 \mathrm{KeV}$ & $100 \mathrm{KeV}$ & $200 \mathrm{KeV}$ & $\begin{array}{c}T \gg m c^{2} \text { (ultra- } \\
\text { relativ.case) }\end{array}$ \\
\hline & 0.97 & 0.73 & 0.61 & 0.47 & $0.25\left(\frac{m c^{2}}{T}\right)^{1 / 2}$ \\
\hline
\end{tabular}

The value of $L$, which enters into the expression for $\chi$ can be written according to (21) as $\left(\right.$ at $\left.\beta=\rho_{i}=4 \cdot 10^{22} \mathrm{~cm}^{-3}\right)$

$$
L=10.8-\frac{3}{2} \ln \frac{m c^{2}}{T}+\ln \bar{x}
$$

where $\bar{x}$ - is some effective value of $x$, corresponding to the maximum of integrand in (53). The magnitudes of $\bar{x}$ and the values of $L$ calculated by the use of (64) are presented in Table 2.

Table 2

\begin{tabular}{r|c|c|c}
\hline$T$ & $50 \mathrm{KeV}$ & $100 \mathrm{KeV}$ & $200 \mathrm{KeV}$ \\
\hline $\bar{x}$ & 10 & 7.5 & 5.8 \\
$L$ & 9.6 & 10.3 & 11.1 \\
\hline
\end{tabular}

In the paper [4] the heat conductivity for nonrelativistic gas was calculated by ChapmanEnskog method. The result was: $\kappa /(1 / L)\left(T / e^{2}\right)^{2}(T / m)^{1 / 2}=0.957$. The difference of this number with the value, presented above is in the limit of the accuracy of both calculations (the accuracy of the result, obtained in [4], is about 2\% - see [5]). ] Note, that the decrease of heat conductivity with increasing of temperature can be expected a priori, since $\chi \sim l v c_{v}$ ( $l$ - is free path length, which is proportional to $E^{2}, c_{v}$ - is the gas specific heat). This estimation results in nonrelativistic case to $T$ dependence $\chi \sim T^{5 / 2}$ and in ultrarelativistic case to $\chi \sim T^{2}$.

\section{The calculation of heat conductivity in the ultrarelativistic case}

\footnotetext{
${ }^{7}$ There is a misprint in the eq.(62) of [4] : it should be $\sqrt{2 \pi}$ instead of $\sqrt{2}$.
} 
Let us consider the case $T \gg m c^{2}$, but suppose, that $T$ is not very large and the movement of ions can be disregarded. In this case the kinetic equation can be obtained by expansion in powers of small parameter $\alpha=m c^{2} / T$. Performing such an expansion in (43) and (45) (evidently one can put $\beta=\gamma=1$ ) we get the equations for distribution functions $\chi_{1}(x)$ and $\chi_{2}(x)$ :

$$
\begin{aligned}
1-\frac{3}{x}=-\frac{1}{x^{2}} \chi_{1}(x) & -\frac{1}{2 x^{2}}\left\{x \chi_{1}(x)-x(4-x) \frac{d \chi_{1}}{d x}-x^{2} \frac{d^{2} \chi_{1}}{d x^{2}}-\right. \\
& \left.-\frac{x}{6} \int_{0}^{\infty} y^{3} e^{-y} \chi_{1}(y) d y\right\} \\
\frac{1}{x}=-\frac{1}{x^{2}} \chi_{2}(x)- & \frac{1}{2 x^{2}}\left\{x \chi_{2}(x)-x(4-x) \frac{d \chi_{2}}{d x}-x^{2} \frac{d^{2} \chi_{2}}{d x^{2}}-\right. \\
& \left.-\frac{x}{6} \int_{0}^{\infty} y^{3} e^{-y} \chi_{2}(y) d y\right\},
\end{aligned}
$$

The heat conductivity is given by:

$$
\kappa=-\frac{1}{24 \pi} \frac{1}{L}\left(\frac{T}{e^{2}}\right)^{2} c \int_{0}^{\infty}\left[\chi_{1}(x)-\zeta \chi_{2}(x)\right] x^{4} e^{-x} d x,
$$

where

$$
\zeta=\frac{\int_{0}^{\infty} e^{-x} x^{3} \chi_{1}(x) d x}{\int_{0}^{\infty} e^{-x} x^{3} \chi_{2}(x) d x} .
$$

Expand $\chi_{1}(x)$ and $\chi_{2}(x)$ over the generalized Laguerre polinomials of the 3-rd power

$$
\begin{aligned}
& \chi_{1}(x)=\sum_{n=0}^{\infty} p_{n} L_{n}(x), \\
& \chi_{2}(x)=\sum_{n=0}^{\infty} q_{n} L_{n}(x),
\end{aligned}
$$

which satisfy the differential equation

$$
x \frac{d^{2} L_{n}(x)}{d x^{2}}+(4-x) \frac{d L_{n}(x)}{d x}+n L_{n}(x)=0
$$

and orthogonality conditions

$$
\int_{0}^{\infty} x^{3} e^{-x} L_{n}(x) L_{m}(x) d x=\delta_{m n} \cdot m !(m+3) !
$$

The substitution of (69) into (65) and the account of (71) gives:

$$
\begin{aligned}
(3-x) x= & \left(1+\frac{x}{2}\right) \sum_{n=0}^{\infty} p_{n} L_{n}(x)+\frac{x}{2} \sum_{n=0}^{\infty} p_{n} L_{n}(x)- \\
& -\frac{x}{12} \sum_{n=0}^{\infty} p_{n} \int_{0}^{\infty} y^{3} e^{-y} L_{n}(y) d y .
\end{aligned}
$$


Multiplay (73) by $x^{3} e^{-x} L_{m}(x)$ and integrate over $x$ in the limits from zero to infinity. Then using the orthogonality condition and the recurrence formula for Laguerre polinomials of the 3-rd power

$$
x L_{n}(x)=2(n+2) L_{n}-L_{n+1}-n(n+3) L_{n-1},
$$

we find the following infinite system of equations for determination of the coefficients $p_{n}$ :

$$
\begin{gathered}
-48 \delta_{n 0}+168 \delta_{n 1}-240 \delta_{n 2}=-2 p_{0}^{\prime}\left(\delta_{n 0}-\delta_{n 1}\right)-\frac{1}{2} n^{2}(n+3) p_{n-1}^{\prime}+ \\
+\left(n^{2}+3 n+3\right) p_{n}^{\prime}-\frac{1}{2}(n+2) p_{n+1}^{\prime} .
\end{gathered}
$$

We introduced the notations $p_{n}^{\prime}=n !(n+3) ! p_{n}$. The equation for determination of $q_{n}^{\prime}$ differs from (75) by its left hand side, which in this case is equal

$$
-24\left(\delta_{n 0}-\delta_{n 1}\right) \text {. }
$$

For the determination of the heat conductivity it is enough to know the zeroth and firsth coefficients of expansion, because

$$
\kappa=-\frac{1}{\pi} \frac{1}{L}\left(\frac{T}{e^{2}}\right)^{2} c\left[p_{0}-p_{1}-\zeta\left(q_{0}-q_{1}\right)\right]
$$

where

$$
\zeta=\frac{p_{0}}{q_{0}}
$$

In order to get the desirable accuracy it is enough to restrict ourself by the account of the first 4 equations in (75) and (76). (The calculation with account of 5 equations demonstrate that the error is less than $2 \%$ in $\kappa$.)

Neglecting $p_{m}^{\prime}$ and $q_{n}^{\prime}$ with $n \geq 5$ and solving the remaining system of equations, we determine

$$
\begin{aligned}
& p_{1}^{\prime}=24, \quad p_{0}^{\prime}=-24 \quad q_{0}^{\prime}=-19.7, \\
& q_{1}^{\prime}=4.32, \\
& p_{1}=1 \quad p_{0}=-4, \quad q_{0}=-3.29 \quad q_{1}=0.18 .
\end{aligned}
$$

By substitution of these values into (77) and (78) we get the final result - the expression for the heat conductivity in the ultrarelativistic case

$$
x=0.25 \frac{1}{L}\left(\frac{T}{e^{2}}\right)^{2} c .
$$

The authors are thankful to A.S.Kronrod for the numerical solution of the equations.

\section{References}

[1] L.D.Landau, Phys.Zs.Sowiet. 10, 1936, 154.

[2] W.Heitler, Quantum theory of radiation, Gostechizdat, 1940.

[3] L.Landau and E.Lifshitz, Statistical physics, Gostechizdat, 1951, p.135.

[4] R.Landshoff, Phys.Rev. 76, 1949, 904.

[5] R.Landshoff, Phys.Rev. 82, 1951, 442. 


\title{
IV To the problem of parity nonconservation in weak interactions 8
}

\author{
B.L.Ioffe, L.B.Okun and A.P.Rudik
}

One of possible explanation of the puzzle, arising in $\theta$ and $\tau$-decays of $K$-mesons [1] is the hypothesis of parity nonconservation in weak interactions. Lee and Yang [2] had shown, that parity nonconservation could not be observed basing on already existing experimental data (except, surely, $K$-mesons decays). They discussed various experiments, which could clear up the problem of parity nonconservation in weak interactions. However, Lee and Yang do not require the invariance of weak interactions relative to time reversal or charge conjugation 9 . If the parity is not conserving ( $\theta$ and $\tau$ are the same particle), then the existence of long living $K^{0}$-meson [4] can be explained by supposing the conservation of the charge parity or the time reversal. The consideration of correlation experiments, performed by Lee and Yang in fact corresponds to supposition of conservation of time parity and violation of charge parity.

If the space parity is not conserving, it is possible to determine experimentally, what parity is conserving - charge parity or time parity. In the case of time parity conservation the longliving (odd under time reversal) $K^{0}$-particle can decay in 3 pions, which are in $S$-state (or $3 \pi^{0}$ ), what is impossible in case of charge parity conservation.

We show below, that the supposition of charge parity conservation results to quite different conclusions, than the ones obtained by Lee and Yang. As is known [3, 5], the invariance of the Hamiltonian under the charge conjugation results to definite phase relations among the coefficients at various interaction terms. Let us consider first the $\Lambda^{0} \rightarrow p+\pi$ decay. If the parity conservation is not required, the interaction Hamiltonian for this process has the form (the terms without derivative are considered):

$$
H=g\left(\bar{\psi}_{p} \psi_{\Lambda}\right) \varphi_{\pi}+i G\left(\bar{\psi}_{p} \gamma_{5} \psi_{\Lambda}\right) \varphi_{\pi}+g^{*}\left(\bar{\psi}_{\lambda} \psi_{p}\right) \varphi_{\pi}^{+}+i G\left(\bar{\psi}_{\Lambda} \gamma_{5} \psi_{p}\right) \varphi_{\pi}^{+} .
$$

The transformation of charge conjugation, as usually, is given by equalities

$$
\varphi^{\prime}(x)=\varphi^{+}(x) ; \quad \psi^{\prime}(x)=-\bar{\psi}(x) C^{-1} ; \quad \bar{\psi}^{\prime}(x)=C \psi(x),
$$

where the matrix $C$ satisfy the conditions

$$
C^{T}=-C ; \quad C C^{+}=1 ; \quad \gamma_{\mu}^{T}=-C \gamma_{\mu} C^{-1}
$$

The Feynman notations are used

$$
\gamma_{\mu}=\{\beta \boldsymbol{\alpha}, \beta\} ; \quad \gamma_{5}=-i \gamma_{1} \gamma_{2} \gamma_{3} \gamma_{4} ; \quad \hat{a}=\gamma_{\mu} a_{\mu}=-\beta \boldsymbol{\alpha} \mathbf{a}+\beta a_{4}
$$

By applying to (1) the charge conjugation transformation and by taken into account that $\gamma_{5}^{T}=C \gamma_{5} C^{-1}$, it is easy to find, that the invariance of the Hamiltonian can be achieved if the constants $g$ and $G$ are real 10

\footnotetext{
${ }^{8} \mathrm{ZhETF}$ 32, 1957, 396.

${ }^{9}$ The general consideration is performed by Pauli [3]. It is important do note, that if parity is not conserved, the charge conjugation is not equivalent to time reversal, since, as was proved by Pauli on the basis of spin and statistic connection, the Lagrangian shall be invariant relative to the product of the transformations of charge conjugation and reflections of all four coordinates.

${ }^{10}$ We suppose, that the spinors, belonging to different fields are anticommuting. The supposition about their commutativity would result to the appearance of nonessential common phase factor.
} 
In the case of parity nonconservation the pseudoscalar terms can contribute to the square of the transition matrix. In order to find out if such terms appear in $\Lambda^{0}$-decay, let us consider the decay of polarized $\Lambda^{0}$ at rest. Using interaction Hamiltonian given by (1) and calculating the square of the matrix element, we find after taken the sum over the proton spin (the proton-pion interaction is not accounted)

$$
\begin{gathered}
\sum|M|^{2}=\left\{g^{2} \bar{u}_{\Lambda}(\hat{p}+m) u_{\Lambda}-G^{2} \bar{u}_{\Lambda} \gamma_{5}(\hat{p}+m) \gamma_{5} u_{\Lambda}+\right. \\
\left.+i g G \bar{u}_{\Lambda}\left[\gamma_{5}(\hat{p}+m)+(\hat{p}+m) \gamma_{5}\right] u_{\Lambda}\right\} / 2 E_{p}
\end{gathered}
$$

where $\hat{p}$ is the momentum and $m$ is the proton mass. The pseudoscalar terms (proportional to $\boldsymbol{\sigma} \mathbf{p}$, where $\boldsymbol{\sigma}$ is $\Lambda^{0}$ spin) could arise evidently from the interference term only. However this term vanishes, since $\bar{u}_{\Lambda} \gamma_{5} u_{\Lambda}=0$. In a similar way it is easy to check, that the terms, proportional to $\boldsymbol{\sigma} \mathbf{p}$, vanish in the case, when persist two types of coupling - the scalar and the vector. From the statement, presented above it follows, that in subsequental processes $\pi^{-}+p \rightarrow \Lambda^{0}+K^{0}, \Lambda^{0} \rightarrow p+\pi^{-}$, considered by Lee and Yang, are absent the pseudoscalar terms (proportional to $\mathbf{p}_{\Lambda} \mathbf{p}_{\pi} \mathbf{p}_{p}$ ), since the role of the first strong interaction process reduces to the creation of polarized $\Lambda^{0}$-particle. Therefore, it is impossible to conclude if the parity is conserved or not in this process by measuring of the proton angular distribution at $\Lambda^{0}$ decay. To distinguish the case of the parity conservation from the case of parity nonservation is possible at radiative $\Lambda^{0}$-decay. In such process the pseudoscalar terms are vanishing in the same way, as above, but the $\gamma$-quantum spectrum will be different, because in the case of parity nonconservation the both types of terms (proportional to $g^{2}$ and $G^{2}$ ) will contribute to the matrix element, what is impossible if parity is conserved.

The other weak process, where the effects, connected with parity nonconservation could arise, is the $\beta$-decay. In the case of parity nonconservation the interaction Hamiltonian has the form

$$
\begin{aligned}
& H=\left(\bar{\psi}_{p} \psi_{n}\right)\left(c_{S} \bar{\psi}_{e} \psi_{\nu}+i c_{S}^{\prime} \bar{\psi}_{e} \gamma_{5} \psi_{\nu}\right)+\left(\bar{\psi}_{p}\left[\gamma_{\mu}, \gamma_{\nu}\right] \psi_{n}\right)\left\{c_{T}\left(\bar{\psi}_{e}\left[\gamma_{\mu}, \gamma_{\nu}\right] \psi_{\nu}\right)+\right. \\
& \left.+i c_{T}^{\prime}\left(\bar{\psi}_{e} \gamma_{5}\left[\gamma_{\mu}, \gamma_{\nu}\right] \psi_{\nu}\right)\right\}+ \text { Herm. conj.; } \quad\left[\gamma_{\mu}, \gamma_{\nu}\right]=1 / 2\left(\gamma_{\mu} \gamma_{\nu}-\gamma_{\nu} \gamma_{\mu}\right)
\end{aligned}
$$

We restrict ourself by consideration of scalar and tensor interactions. The existing experiments indicate, that just these variants are realized. The use of charge conjugation invariance gives that the constants $c_{S}, c_{S}^{\prime}, c_{T}, c_{T}^{\prime}$ are real.

Consider the $\beta$-decay of polarized nucleons. Calculate, using (3), the square of the matrix element and perform the summation over the spins of neutrino. It is easy to convince yourself, that among the pseudoscalar terms remains only the terms, arising from the interference of the scalar and tensor variants, while the terms, proportional to $c_{S} c_{S}^{\prime}$ and $c_{T} c_{T}^{\prime}$ are identically zero. After summing over electron spin we get ( $\mathbf{q}$ is the neutrino momentum):

$$
\begin{gathered}
\left(4 / E_{\nu} E_{e}\right)\left(c_{S}^{\prime} c_{T}-c_{S} c_{T}^{\prime}\right)\left\{\operatorname { I m } \left(\int \bar{\psi}_{p} \psi_{n} \exp \left[-i\left(\mathbf{p}_{e}-\mathbf{q}\right) \mathbf{r}\right] d \mathbf{r} \times\right.\right. \\
\left.\times \int \bar{\psi}_{n} \boldsymbol{\sigma} \psi_{p} \exp \left[i\left(\mathbf{p}_{e}-\mathbf{q}\right) \mathbf{r}\right] d \mathbf{r}\right)\left(\mathbf{q} E_{e}-\mathbf{p} E_{\nu}\right)+ \\
\left.+\operatorname{Re}\left(\int \bar{\psi}_{p} \psi_{n} \exp \left[-i\left(\mathbf{p}_{e}-\mathbf{q}\right) \mathbf{r}\right] d \mathbf{r} \int \bar{\psi}_{n} \boldsymbol{\alpha} \psi_{p} \exp \left[i\left(\mathbf{p}_{e}-\mathbf{q}\right) \mathbf{r}\right] d \mathbf{r}\right)[\mathbf{p q}]\right\} .
\end{gathered}
$$

The expression (4) can be easy calculated in the case of polarized neutron $\beta$-decay and is equal to zero in the case, when the proton polarization is not measured and the sum over its 
spin is performed. Expression (4) vanishes also in the case of allowed transitions. Indeed, at the allowed transitions only the first term in the figure brackets is accounted in (4) and the $\exp \left[i\left(\mathbf{p}_{e}-\mathbf{q}\right) \mathbf{r}\right]$ can be replaced by 1 . Then, because of the presence of scalar interaction the initial and final states correspond to the same value of the total momentum and its projection. But, as it is known, the diagonal matrix elements of the Hermitian operator $\boldsymbol{\sigma}$ are real, what results to vanishing of (44). For the allowed transitions (44) is vanishing if it is accounted the interaction of the electron with the Coulomb field of nucleons (in the first approximation in $Z e / \hbar v)$.

Therefore, the requirement of charge conjugation invariance, results to the absence of differences between the cases of parity conservation and nonconservation also in the simplest situations in $\beta$-decay.

The authors are very indepted to Prof.V.B.Berestetsky and V.V.Sudakov for discussion and to Prof.I.Ya.Pomeranchuk for his interest to our work.

Received by the editors at 21.11.1956

Note added in proof (14.02.1957). Quite recently became known the results of experiments done by Prof. Wu on the $\beta$-decay of oriented nucleous $C o$. In these experiments was found the correlation of nuclear spin and direction of electron momentum (the term $\boldsymbol{\sigma} \mathbf{p}$ ). In accord with presented above statements this fact indicates on nonconservation of space and charge parities in $\beta$-decay.

\section{References}

[1] J.Orear, G.Harris, S.Taylor, Phys.Rev. 102, 1976, 1956.

[2] T.D.Lee, C.N.Yang (preprint).

[3] W.Pauli, Niels Bohr and the development of physics. Pergamon Press, London, 1955.

[4] K.Lande, E.T.Booth, J.Impeduglia, L.M.Lederman, W.Chinowsky, Phys. Rev. 103, 1956, 1900; W.Fry, J.Schneps, M.S.Swami, Phys.Rev. 103, 1956, 1904.

[5] H.A.Tolhoek, S.R.DeGroot, Phys.Rev. 84, 1951, 150. 


\title{
$\mathrm{V}$ On the two possible schemes of parity nonconservation in weak interactions 11
}

\author{
B.L.Ioffe
}

One of the possible explanations of $K^{+}$- mesons decay into two and tree $\pi$-mesons is the hypothesis of parity nonconservation in weak interactions [1]. If this hypothesis is accepted, then the question arises: if it is conserved the charge parity and the parity relative to time inversion in weak interaction. As is known [2], the connection of spin with statistic requires the invariance of all interactions under the product of three transformations: the reflection of three space coordinate $I$, the time inversion $T$ and charge conjugation $C$, symbolically $I T C=1$. Therefore [3], in the case of parity violation in weak interactions $(I \neq 1)$ there are three possibilities : I) weak interactions are invariant under time reversal $(T=1)$, but not invariant under charge conjugation and $I C=1$; II) weak interactions are invariant under charge conjugation $(C=1)$, but not invariant under time reversal and $I T=1$; III) weak interactions are noninvariant under charge conjugation as well as under time reversal, but $I T C=1$. It the last possibility is accepted, than the fact of the existence of long lived $K^{0}$ [4] looks as a pure accident, since the Gell-Mann and Pais arguments [5], which are the base of this prediction holds only in the case of conservation of charge parity or the parity under time reversal. This fact allows to throw away the last possibility and to consider the first two only.

In this paper we consider, what physical phenomena can take place in each of these alternative possibilities. The first of these possibilities, as was mentioned by Landau[6], physically corresponds to the supposition, that all interactions are invariant under simultaneous change of left to right and to transfer from particles to antiparticles. The physical sense of the second supposition is that all interactions are invariant if simultaneously with the transfer from left to right proceeds the backward movement in time.

Consider first the scheme I, when at the parity violation is conserved the time inversion. Let at $t \rightarrow-\infty$ exist the set of particles in the state $a$, the momenta in this state are $\mathbf{p}_{a}$ and the mean values of spins are $\mathbf{s}_{a}$. As a result of interaction this system of particles at $t \rightarrow+\infty$ transfers into the other system with momenta $\mathbf{p}_{b}$ and mean values of spins $\mathbf{s}_{b}$. From the time reversal invariance than follows [7] that the matrix element of the transition $S_{a b}^{I}\left(\mathbf{p}_{a}, \mathbf{s}_{a} ; \mathbf{p}_{b}, \mathbf{s}_{b}\right)$ is connected with the matrix element of the inverse transition $S_{b a}^{I}\left(\mathbf{p}_{b}, \mathbf{s}_{b} ; \mathbf{p}_{a}, \mathbf{s}_{a}\right)$ by

$$
S_{a b}^{I}\left(\mathbf{p}_{a}, \mathbf{s}_{a} ; \mathbf{p}_{b}, \mathbf{s}_{b}\right)=S_{b a}^{I}\left(-\mathbf{p}_{b}-\mathbf{s}_{b} ;-\mathbf{p}_{a},-\mathbf{s}_{a}\right) .
$$

The matrix element $S_{b a}$, considered as a function of its arguments, do not have, generally, the same functional form as the function $S_{a b}$. This circumstance do not allow to have a direct profit from the relation (11). However, if the transition $a \rightarrow b$ proceeds due to weak interaction, then in the first nonvanishing approximation in this interaction the detailed balance relation takes place

$$
S_{a b}\left(\mathbf{p}_{a}, \mathbf{s}_{a} ; \mathbf{p}_{b}, \mathbf{s}_{b}\right)=-S_{b a}^{*}\left(\mathbf{p}_{b}, \mathbf{s}_{b} ; \mathbf{p}_{a}, \mathbf{s}_{a}\right)
$$

(The relation (2) is legitimate if transition proceeds because of weak interaction, but it is not required that the movement of the particle is described by free wave functions.) By

${ }^{11}$ ZhETF 32, 1957, 1246. 
excluding $S_{b a}$ from (11) and (2) we find that in the case of the invariance under time reversal the transition matrix element satisfies the equality

$$
S_{a b}^{I}\left(\mathbf{p}_{a}, \mathbf{s}_{a} ; \mathbf{p}_{b}, \mathbf{s}_{b}\right)=-S_{a b}^{I *}\left(-\mathbf{p}_{a},-\mathbf{s}_{a} ;-\mathbf{p}_{b},-\mathbf{s}_{b}\right)
$$

and. consequently, the probability of the transition satisfies the relation

$$
W_{a b}^{I}\left(\mathbf{p}_{a}, \mathbf{s}_{a} ; \mathbf{p}_{b}, \mathbf{s}_{b}\right)=W_{a b}^{I}\left(-\mathbf{p}_{a},-\mathbf{s}_{a} ;-\mathbf{p}_{b},-\mathbf{s}_{b}\right) .
$$

The analogous consideration can be performed in the case of the second possible scheme, when all interactions are invariant under charge conjugation. In this case instead of time inversal transformation it is necessary to consider the inversion of all four coordinates. The matrix elements of the direct and inversed transitions are connected by the relation

$$
S_{a b}^{I I}\left(\mathbf{p}_{a}, \mathbf{s}_{a} ; \mathbf{p}_{b}, \mathbf{s}_{b}\right)=S_{b a}^{I I}\left(\mathbf{p}_{b},-\mathbf{s}_{b} ; \mathbf{p}_{a},-\mathbf{s}_{a}\right) .
$$

The equality (2), which is based on the Hermitancy of Hamiltonian is valid in this case also. The substitution of (2) into (4) gives

$$
S_{a b}^{I I}\left(\mathbf{p}_{a}, \mathbf{s}_{a} ; \mathbf{p}_{b}, \mathbf{s}_{b}\right)=-S_{a b}^{I I *}\left(\mathbf{p}_{a},-\mathbf{s}_{a} ; \mathbf{p}_{b},-\mathbf{s}_{b}\right) .
$$

Therefore, in the case of the scheme II the transition probability satisfy the relation

$$
W_{a b}^{I I}\left(\mathbf{p}_{a}, \mathbf{s}_{a} ; \mathbf{p}_{b}, \mathbf{s}_{b}\right)=W_{a b}^{I I}\left(\mathbf{p}_{a},-\mathbf{s}_{a} ; \mathbf{p}_{b},-\mathbf{s}_{b}\right) .
$$

By the use (A) and (B) it is easy to determine the general form of the transition probability for both schemes of parity nonconservation.

Consider, for example, the decay of polarized $\Lambda$-particle at rest: $\Lambda^{0} \rightarrow p+\pi^{-}$. In general, $\Lambda$ decay is characterized by three vectors: the $\Lambda$ spin $\mathbf{s}_{\Lambda}$, proton momentum $\mathbf{p}_{p}$ and proton spin $\mathbf{s}_{\beta}$. We are interesting by pseudoscalar quantities, arising from parity nonconservbation. From three vectors it is possible to constract three such expressions $\mathbf{s}_{p} \mathbf{p}_{p}, \mathbf{s}_{\Lambda} \mathbf{p}_{p},\left[\mathbf{s}_{\Lambda} \mathbf{s}_{p}\right] \mathbf{p}_{p}$. From (A) and (B) it follows that in the case of scheme $I$ the probability can contain the terms $\mathbf{s}_{p} \mathbf{p}_{p}$ and $\mathbf{s}_{\Lambda} \mathbf{p}_{p}$, whereas in the case of the scheme $I I$ in the probability only the terms, proportional to $\left[\mathbf{s}_{\Lambda} \mathbf{s}_{p}\right] \mathbf{p}_{p}$ can appear. So, in the scheme I at $\Lambda$ decay one can expect the proton polarization along (or opposite) the direction of proton momentum, or if $\Lambda$-particle is polarized the protons will be mainly directed along (or opposite) the $\Lambda$ spin. (This effect was considered by Lee and Yang[1].) In the scheme II the effect of parity nonconservation can be found only by observation of proton polarization at the decay of polarized $\Lambda$. Or, what is equivalent, by the measurement of the directions of proton spin and momentum relative to the normal to the plane of $\Lambda$ production. In a similar way it is possible to find the pseudoscalar terms allowed in other weak decays for each scheme. The results are presented in the Table. (The indexes $\Lambda, p, e, \mu$ are related to the spins and momenta of $\Lambda$, proton, electron and $\mu$-meson correspondingly; in the case of $\beta$-decay: $\mathbf{I}_{N}$ - is the spin of initial nucleous, $\mathbf{p}_{N}$ - is the momentum of recoil nucleous, the averaging is performed over all other momenta.) 


\section{Table}

\begin{tabular}{c|c|c}
\hline $\begin{array}{c}\text { The type } \\
\text { of decay }\end{array}$ & $\begin{array}{c}\text { Scheme I } \\
T=i n v\end{array}$ & $\begin{array}{c}\text { Scheme II } \\
C=i n v\end{array}$ \\
\hline$\Lambda$ & $\mathbf{s}_{p} \mathbf{p}_{p} ; \mathbf{s}_{\Lambda} \mathbf{p}_{p}$ & {$\left[\mathbf{s}_{\Lambda} \mathbf{s}_{p}\right] \mathbf{p}_{p}$} \\
$\beta$ & $\mathbf{s}_{e} \mathbf{p}_{e} ; \mathbf{s}_{e} \mathbf{p}_{N}$ & {$\left[\mathbf{s}_{e} \mathbf{I}_{N}\right] \mathbf{p}_{e}$} \\
& $\mathbf{I}_{N} \mathbf{p}_{e} ; \mathbf{I}_{N} \mathbf{p}_{N}$ & {$\left[s_{e} \mathbf{I}_{N}\right] \mathbf{p}_{N}$} \\
$\pi$ & $\mathbf{s}_{\mu} \mathbf{p}_{\mu}$ & - \\
$\mu$ & & \\
& $\mathbf{s}_{\mu} \mathbf{p}_{e} ; \mathbf{s}_{e} \mathbf{p}_{e}$ & {$\left[\mathbf{s}_{\mu} \mathbf{s}_{e}\right] \mathbf{p}_{e}$} \\
\hline
\end{tabular}

The nucleous dipole momenta are absent in the scheme I [6], but could persist in the scheme II. Indeed, the energy of interaction of the dipole momentum with electric field is proportional to $\mathbf{s E}$. At the time reflection $\mathbf{s} \rightarrow-\mathbf{S}$ and $\mathbf{E} \rightarrow \mathbf{E}$, at the reflection of all four coordinates: $\mathbf{s} \rightarrow-\mathbf{s}$ and $\mathbf{E} \rightarrow-\mathbf{E}$. Therefore, $\mathbf{s E} \rightarrow-\mathbf{s E}$ in the scheme I and $\mathbf{s E} \rightarrow \mathbf{s E}$ in the scheme II.

The author is thankful to L.B.Okun and A.P.Rudik for very useful discussions as well to L.D.Landau for encouragement.

Received by the editors at 19.01.1957

\section{References}

[1] T.D.Lee, C.N.Yang, Phys.Rev. 104, 1956, 254.

[2] W.Pauli, Niels Bohr and the development of physics, Pergamon Press, London, 1955.

[3] B.L.Ioffe, L.B.Okun, A.P.Rudik, ZhETF 32, 1957, 396.

[4] R.Lande et al. Phys.Rev. 103, 1956, 1900. W.Fry et al. Phys.Rev.103 (1956) 1904.

[5] M.Gell-Mann, A.Pais, Phys.Rev. 97, 1955, 1387.

[6] L.D.Landau, ZhETF 32, 1957, 405.

[7] J.Blatt, V.Weisskopf, Theoretical Nuclear Physics, New York-London, 1952. 